\title{
ESTIMATION OF HARMONIC COMPONENT IN REGRESSION WITH CYCLICALLY DEPENDENT ERRORS
}

\author{
Ivanov, A.V. ${ }^{1}$, Leonenko, N.N. ${ }^{2}$, Ruiz-Medina, M.D. ${ }^{3}$ and Zhurakovsky, B.M. ${ }^{1}$
}

June 20, 2018

\begin{abstract}
This paper deals with the estimation of hidden periodicities in a non-linear regression model with stationary noise displaying cyclical dependence. Consistency and asymptotic normality are established for the least-squares estimates.

1 National Technical University "Kyiv Polytechnic Institute"

37 Peremogy Ave, 03056 Kyiv, Ukraine

${ }^{2}$ School of Mathematics, Cardiff University,

Senghennydd Road, Cardiff CF24 4YH, United Kingdom

${ }^{3}$ Department of Statistics and Operations Research

University of Granada, Campus Fuente Nueva s/n, E-18071 Granada, Spain
\end{abstract}

Keywords: Asymptotic distribution theory, asymptotic inference, hidden periodicities, nonlinear regression, vector parameter.

AMS classifications: $62 \mathrm{E} 20 ; 62 \mathrm{~F} 10 ; 60 \mathrm{G} 18$

\section{Introduction}

Let us consider the regression model

$$
x(t)=g(t, \theta)+\varepsilon(t),
$$

where $g(t, \theta): \mathbb{R} \times \Theta^{c} \rightarrow \mathbb{R}$ is a continuous non-linear function of unknown parameter vector $\theta \in \Theta$, $\theta=\left(\theta^{1}, \ldots, \theta^{q}\right)$, with $\Theta^{c}$ being the closure in $\mathbb{R}^{q}$ of open set $\Theta \subset \mathbb{R}^{q}$, and $\{\varepsilon(t), t \in \mathbb{R}\}$ is the random noise process defining the error term through time. Process $\varepsilon$ is assumed to be a zeromean stationary process, generated by non-linear transformation of a stationary Gaussian process $\xi$ displaying cyclical dependence. We address the problem of the estimation of the unknown parameter $\theta$ from the observation of random process $\{x(t), t \in[0, T]\}$, when $T \rightarrow \infty$.

The least-squares estimate (LSE) $\hat{\theta}_{T}$ of an unknown parameter $\theta \in \Theta$, obtained from the observations $\{x(t), t \in[0, T]\}$, is any random variable $\hat{\theta}_{T} \in \Theta^{c}$, having the property

$$
Q_{T}\left(\hat{\theta}_{T}\right)=\inf _{\tau \in \Theta^{c}} Q_{T}(\tau), \quad Q_{T}(\tau)=\frac{1}{T} \int_{0}^{T}[x(t)-g(t, \tau)]^{2} d t,
$$

where $\Theta^{c}$ is the closure of $\Theta$. 
Our main interest in this paper is the problem of detecting hidden periodicities, that is, the problem of estimation of the unknown parameters of the regression function

$$
g(t, \theta)=\sum_{k=1}^{N}\left(A_{k} \cos \varphi_{k} t+B_{k} \sin \varphi_{k} t\right),
$$

where $\theta=\left(\theta_{1}, \theta_{2}, \theta_{3}, \ldots, \theta_{3 N-2}, \theta_{3 N-1}, \theta_{3 N}\right)=\left(A_{1}, B_{1}, \varphi_{1}, \ldots, A_{N}, B_{N}, \varphi_{N}\right) \in \mathbb{R}^{3 N}, C_{k}^{2}=A_{k}^{2}+B_{k}^{2}>$ $0, k=1, \ldots, N, \quad 0 \leq \varphi<\varphi_{1}<\cdots<\varphi_{N}<\bar{\varphi}<\infty$.

This paper provides the asymptotic properties of the LSE of the nonlinear regression model (1) with regression function (3) and cyclical dependent stationary noise. Specifically, the consistency and the convergence to the Gaussian distribution of the LSE of the parameters involved in the definition of the regression function (3) are derived in this paper.

Although in the subsequent development, we will refer to the nonlinear regression model (11) with regression function (31), and cyclical dependent stationary noise with covariance function (6), the results given in Section 4 and 5 on linearization, and asymptotic uniqueness, as well as on asymptotic normality hold for a more general class of regression functions satisfying conditions B1-B6 formulated below. The general class of non-linear regression functions that could be considered includes the family of functions $g$ whose matrix-valued measure, defined for $T>0$ by,

$$
\begin{gathered}
\boldsymbol{\mu}_{T}(d \lambda)=\left(\mu_{T}^{j l}(d \lambda, \theta)\right)_{j, l=1}^{q}, \quad \mu_{T}^{j l}(d \lambda, \theta)=\frac{\left.g_{T}^{j}(\lambda, \theta) \overline{g_{T}^{l}(\lambda, \theta)}\right) d \lambda}{\left(\int_{\mathbb{R}}\left|g_{T}^{j}(\lambda, \theta)\right|^{2} d \lambda \int_{\mathbb{R}}\left|g_{T}^{l}(\lambda, \theta)\right|^{2} d \lambda\right)^{\frac{1}{2}}}, \quad j, l=1, \ldots, q, \\
g_{T}^{j}(\lambda, \theta)=\int_{0}^{T} e^{i t \lambda} \frac{\partial}{\partial \theta_{j}} g(t, \theta) d t, \quad j=1, \ldots, q, \quad \lambda \in \mathbb{R}, \quad \theta \in \Theta,
\end{gathered}
$$

could weakly converge as $T \rightarrow \infty$ to an atomic spectral measure $\boldsymbol{\mu}$ with atoms $\Xi_{\text {regr }}=\left\{\delta_{1}, \ldots, \delta_{n}\right\}$. Limit theorems for non-linear transformations of Gaussian stationary processes are here considered. In the derivation of these limit results, the mentioned weak-convergence to the spectral measure associated with the regression function, and the diagram formulae are applied. In the discrete case this phenomenon was discussed by Yajima $(1988,1991)$ in some other regression scheme.

Note that the classical non-central limit theorems (Taqqu, 1979, and Dobrushin and Major, 1979) can be viewed as particular cases of the general setting considered here, when the noise is the non-linear transformation of a Gaussian process with the unique singular point in the spectrum: $\Xi_{\text {noise }}=\{0\}$, and the regression function is such that $\frac{\partial}{\partial \theta} g(t, \theta) \equiv 1(q=1)$. In this case, the regression measure $\mu_{T}$ is the Fejer kernel, which tends to the delta-measure with atom at zero, that is, the limit $\mu$-measure spectrum consists of one point: $\Xi_{\text {regr }}=\{0\}$. Nonstandard renormalizations and special limiting distributions are required here due to the fact that $\Xi_{\text {noise }} \cap \Xi_{\text {regr }} \neq \emptyset$. Some limiting distributions for the case where the two spectral point sets $\Xi_{\text {noise }}$ and $\Xi_{\text {regr }}$ are in fact overlapped, in the discrete case, can be derived from the papers by Taqqu (1975, 1979), Rosenblatt (1981, 1987), Viano et al (1995), Oppenheim et al (2002), Haye (2002), Haye and Viano (2002), Haye and Phillipe (2003), Arcones $(1994,2000)$. In the continuous time case, the limiting distributions for non-empty set $\Xi_{\text {noise }} \cap \Xi_{\text {regr }}$ can be obtained from the papers and book by Ivanov and Leonenko (1989, 2004, 2008), and Leonenko and Taufer (2006). For the non-linear regression model with function $g$ given by (3), this subject will be considered in subsequent papers. In the present paper we consider the case when $\Xi_{\text {noise }} \cap \Xi_{\text {regr }}=\emptyset$. This assumption as it will be seen leads to the asymptotic normality of the LSE of parameters of model (3).

During the last thirty years, a number of papers have been devoted to limit theorems for non-linear transformations of Gaussian processes and random fields. The pioneer results are those of Taqqu (1975, 1979) and Dobrushin and Major (1979) for convergence to Gaussian and non-Gaussian distributions under long range dependence in terms of Hermite expansions, and Breuer and Major (1983), Avram and Brown (1989), Ivanov and Leonenko (1989), Avram (1992), Avram and Fox (1992) for convergence to Gaussian limit distribution by using diagram formulae or graphical methods. This line of research continues to be of interest today, see Berman (1992) for $m$-dependent approximation approach, Ho and Hsing (1997) for martingale approach, Nualart and Pecatti (2005) (see also Pecatti and Tudor 
(2004)) for using Malliavin calculus, Avram, Leonenko and Sakhno (2010) for an extension of graphical method for random fields, to name only a few papers. The volume of Doukhan, Oppenheim and Taqqu (2003) contains outstanding surveys of the field. In particular, that volume discusses different definitions of short range dependence and long range dependence of stationary processes in terms of the autocorrelation function (the integral of the correlation function diverges) or the spectrum (the spectral density has a singularity at zero).

Non-linear regression models with independent or weakly dependent errors have been extensively studied (see, for example, Hannan (1973), Ivanov and Leonenko (1989), Ivanov (1997), Skouras (2000), Polard and Radchenko (2006) and the references therein). The first results on non-linear regression with long-range dependence (LRD) were obtained by Robinson and Hidalgo (1997). They established conditions for consistency of some estimates of a parameter of non-linear regression with LRD errors in discrete time models. Important results on asymptotic distribution of M-estimators in non-linear regression models with discrete time and LRD property of the noise process are presented in Koul and Baillie (2003), and Koul (1996) papers, both, for smooth and more general score functions.

Note that as we have considered the case of continuous time regression, where the response variables are observed over continuous time, parameters characterizing local regularity properties, such as parameter of intermittency, for example, in the fractional Riesz-Bessel motion model, can be estimated in this setting (see, for example, Avram, Leonenko and Sakhno (2010)).

The asymptotic theory of LSE in non-linear regression with LRD has been considered by Mukhergee (2000) and Ivanov and Leonenko (2004, 2008). The papers by Ivanov and Leonenko (2009) and Ivanov and Orlovsky (2008) discuss the asymptotic distributions of a class of M-estimates and $\mathrm{L}_{p}$-estimates $(1<p<2)$ in the nonlinear regression model with LRD. Our paper is a continuation of these papers.

The problem of the estimation of the parameters characterizing the distribution of the noise is not addressed here. This will be the subject of subsequent papers in the spirit of the paper by Ivanov and Leonenko (2008).

\section{Stationary processes with cyclical dependence}

We recall the assumptions that will be made on the Gaussian process $\xi$ generating the random noise $\varepsilon$, representing the time-dependent error term in the regression model (10). Specifically, we will consider a stationary process $\xi$ in continuous time defined on a complete probability space $(\Omega, \mathbb{F}, P)$ :

$$
\xi(t)=\xi(\omega, t): \Omega \times \mathbb{R} \longrightarrow \mathbb{R},
$$

satisfying the following assumption.

A1. Random function $\xi=\{\xi(t), t \in \mathbb{R}\}$ is a real-valued and measurable stationary mean-square continuous Gaussian process with $E \xi(t)=0$, and $E \xi^{2}(t)=1$. Its covariance function (c.f.) is of the form:

$$
B(t)=E[\xi(0) \xi(t)]=\sum_{j=0}^{\kappa} D_{j} B_{\alpha_{j}, \varkappa_{j}}(t), t \in \mathbb{R}, \kappa \geq 0, \quad \sum_{j=0}^{\kappa} D_{j}=1, D_{j} \geq 0, j=0, \ldots, \kappa,
$$

where

$$
B_{\alpha_{j}, \varkappa_{j}}(t)=\frac{\cos \left(\varkappa_{j} t\right)}{\left(1+t^{2}\right)^{\alpha_{j} / 2}}, \quad 0 \leq \varkappa_{0}<\varkappa_{1}<\ldots<\varkappa_{\kappa}, \quad \alpha_{j}>0, \quad t \in \mathbb{R}, \quad j=0, \ldots, \kappa,
$$

Remark 1 If $\varkappa_{0}=0$ and $0<\alpha_{0}<1$, process $\xi$ displays long-range dependence. Otherwise, process $\xi$ is of short range dependence.

The c.f. $B(t), t \in \mathbb{R}$, admits the following spectral representation:

$$
B(t)=\int_{\mathbb{R}} e^{i \lambda t} f(\lambda) d \lambda, \quad t \in \mathbb{R}
$$


where the spectral density (s.d.) is of the form:

$$
f(\lambda)=\sum_{j=0}^{\kappa} D_{j} f_{\alpha_{j}, \varkappa_{j}}(\lambda), \quad \lambda \in \mathbb{R}
$$

with, for $j=0, \ldots, \kappa, f_{\alpha_{j}, \varkappa_{j}}(\lambda)$ being defined by

$$
f_{\alpha_{j}, \varkappa_{j}}(\lambda)=\frac{c_{1}\left(\alpha_{j}\right)}{2}\left[K_{\frac{\alpha_{j}-1}{2}}\left(\left|\lambda+\varkappa_{j}\right|\right)\left|\lambda+\varkappa_{j}\right|^{\frac{\alpha_{j}-1}{2}}+K_{\frac{\alpha_{j}-1}{2}}\left(\left|\lambda-\varkappa_{j}\right|\right)\left|\lambda-\varkappa_{j}\right|^{\frac{\alpha_{j}-1}{2}}\right], \quad \lambda \in \mathbb{R},
$$

and

$$
c_{1}\left(\alpha_{j}\right)=\frac{2^{\left(1-\alpha_{j}\right) / 2}}{\sqrt{\pi} \Gamma\left(\frac{\alpha_{j}}{2}\right)} .
$$

Here,

$$
K_{\nu}(z)=\frac{1}{2} \int_{0}^{\infty} s^{\nu-1} \exp \left\{-\frac{1}{2}\left(s+\frac{1}{s}\right) z\right\} d s, \quad z \geq 0, \quad \nu \in \mathbb{R},
$$

is the modified Bessel function of the third kind and order $\nu$ or McDonald's function.

The following derived identities constitute an improvement and correction of Anh, Knopova and Leonenko (2004). Indeed, we omit some details. For a small $z$, the following asymptotic expansions are known (see, i.e., Gradshteyn and Ruzhik (2000), formulae 8.825, 8.445 and 8.446): if $\nu \notin \mathbb{Z}$,

$$
\begin{gathered}
K_{-\nu}(z)=K_{\nu}(z)= \\
=\frac{\pi}{2 \sin (\pi \nu)}\left\{\sum_{j=0}^{\infty} \frac{(z / 2)^{2 j-\nu}}{j ! \Gamma(j+1-\nu)}-\sum_{j=0}^{\infty} \frac{(z / 2)^{2 j-\nu}}{j ! \Gamma(j+1+\nu)}\right\},
\end{gathered}
$$

while if $\nu= \pm m$, where $m$ is a nonegative integer,

$$
\begin{gathered}
K_{\nu}(z)=\frac{1}{2} \sum_{j=0}^{m-1} \frac{(-1)^{j}(m-j-1) !}{j !}\left(\frac{z}{2}\right)^{2 j-m}+ \\
+(-1)^{m+1} \sum_{j=0}^{\infty} \frac{(z / 2)^{m+2 j}}{j !(m+j) !}\left\{\ln \frac{z}{2}-\frac{1}{2} \Psi(j+1)-\frac{1}{2} \Psi(j+m+1)\right\},
\end{gathered}
$$

where $\Psi(z)=\left(\frac{d}{d z} \Gamma(z)\right) / \Gamma(z)$ is the logarithm derivative of the Gamma function.

Therefore we have: for $\alpha_{j}>1$

$$
\lim _{\lambda \rightarrow 0} f_{\alpha_{j}, 0}(\lambda)=\frac{\Gamma\left(\frac{\alpha_{j}-1}{2}\right)}{\left[2 \sqrt{\pi} \Gamma\left(\frac{\alpha_{j}}{2}\right)\right]},
$$

for $\alpha_{j}=1$, and $\lambda \rightarrow 0$

$$
f_{1,0}(\lambda) \sim \frac{1}{\pi}\{-\ln |\lambda|+\ln 2+\Psi(1)\},
$$

where $\Psi(1)=-\gamma, \gamma$ is the Euler constant.

For $0<\alpha_{j}<1$, and $\lambda \rightarrow 0$

$$
f_{\alpha_{j}, 0}(\lambda)=c_{2}\left(\alpha_{j}\right) \frac{1}{|\lambda|^{1-\alpha_{j}}}\left(1-h_{j}(|\lambda|)\right),
$$

where $c_{2}\left(\alpha_{j}\right)=\left[2 \Gamma\left(\alpha_{j}\right) \cos \frac{\alpha_{j} \pi}{2}\right]^{-1}$, and $\left|h_{j}(|\lambda|)\right|<1$.

Thus, for $j=0, \ldots, \kappa, 0<\alpha_{j}<1$, in the neighborhood of the points $\varkappa_{j}$ :

$$
f_{\alpha_{j}, \varkappa_{j}}(\lambda)=c_{2}\left(\alpha_{j}\right)\left[\left|\lambda+\varkappa_{j}\right|^{\alpha_{j}-1}\left(1-h_{j}\left(\left|\lambda+\varkappa_{j}\right|\right)\right)\right] .
$$


Therefore, the s.d. $f$ has $2 \kappa+2$ different singular points $\left\{-\varkappa_{\kappa},-\varkappa_{\kappa-1}, . .,-\varkappa_{1},-\varkappa_{0}, \varkappa_{0}, \varkappa_{1}, \ldots, \varkappa_{\kappa}\right\}$ under condition $\mathbf{A} 1$, when $\varkappa_{0} \neq 0$, and $0<\alpha_{j}<1, j=0, \ldots, \kappa$. If $\varkappa_{0}=0$, the s.d. $f$ has $2 \kappa+1$ different singular points.

For $\alpha_{j}=1$ and $\lambda \rightarrow \pm \varkappa_{j}$ :

$$
f_{1, \varkappa_{j}}(\lambda) \sim \frac{c_{1}\left(\alpha_{j}\right)}{2} K_{0}\left(\left|2 \varkappa_{j}\right|\right)+\frac{1}{2} \frac{1}{\pi}\left\{-\ln \left|\lambda \mp \varkappa_{j}\right|+\ln 2+\Psi(1)\right\}
$$

while for $\alpha_{j}>1$ and $\lambda \rightarrow \pm \varkappa_{j}$ :

$$
f_{\alpha_{j}, \varkappa_{j}}(\lambda) \rightarrow \frac{c_{1}\left(\alpha_{j}\right)}{2} K_{\frac{\alpha_{j}-1}{2}}\left(\left|2 \varkappa_{j}\right|\right)+\frac{1}{2} \frac{\Gamma\left(\frac{\alpha_{j}-1}{2}\right)}{\left[2 \sqrt{\pi} \Gamma\left(\frac{\alpha_{j}}{2}\right)\right]} .
$$

Similar results can be obtained for c.f.'s defined as linear combinations of the functions

$$
R_{\alpha_{j}, \varkappa_{j}}(t)=\frac{\cos \left(\varkappa_{j} t\right)}{\left(1+|t|^{\rho_{j}}\right)^{\alpha j}}, \quad \varkappa_{j} \in \mathbb{R}, \quad 0<\rho_{j} \leq 2, \quad \alpha_{j}>0, \varkappa_{j} \neq 0, \quad j=0, \ldots, \kappa
$$

(see again Ivanov and Leonenko, 2004, and Anh, Knopova and Leonenko, 2004, for details, also some formulae of the last paper have been corrected in the text).

\section{Consistency}

This section is devoted to the derivation of the weak-consistency of the LSE

parameter estimator, in the Walker sense. Some additional conditions are first formulated, needed in the subsequent results.

A2. The stochastic process $\varepsilon$ is given by $\varepsilon(t)=G(\xi(t)), t \in \mathbb{R}$, with $\xi(t)$ satisfying condition A1, and $G: \mathbb{R} \longrightarrow \mathbb{R}$ being a non-random measurable function such that $E G(\xi(0))=0$, and $E G^{4}(\xi(0))<$ $\infty$.

Under condition A2, function $G \in L_{2}(\mathbb{R}, \varphi(x) d x)$, with $\varphi(x)=\frac{1}{\sqrt{2 \pi}} e^{-\frac{x^{2}}{2}}, x \in \mathbb{R}$, being the standard Gaussian density, and

$$
G(x)=\sum_{k=1}^{\infty} \frac{C_{k}}{k !} H_{k}(x), \quad \sum_{k=1}^{\infty} \frac{C_{k}^{2}}{k !}=E\left[G^{2}(\xi(0))\right]<\infty,
$$

where

$$
C_{k}=\int_{\mathbb{R}} G(x) H_{k}(x) \varphi(x) d x
$$

Here, the Hermite polynomials

$$
H_{k}(x)=(-1)^{k}[\sqrt{2 \pi} \varphi(x)]^{-1} \frac{d^{k}}{d x^{k}}[\sqrt{2 \pi} \varphi(x)], \quad k=0,1,2, \ldots,
$$

constitute a complete orthogonal system in the Hilbert space $L_{2}(\mathbb{R}, \varphi(x) d x)$.

Remark 2 Denoting by $\phi$ the distribution function (d.f.) of the standard normal distribution, it is easy to see that the process $\varepsilon(t)=G(\xi(t))=F^{-1}(\phi(\xi(t)))$ has a marginal d.f. F for any strictly increasing d.f. with zero mean. Thus, we can introduce regression models with Student errors, for example.

A3. We assume that the function $G$ has Hermite $\operatorname{rank} \operatorname{Hrank}(G)=m$, that is, either $C_{1} \neq 0$ and $m=1$, or, for some $m \geq 2, C_{1}=\cdots=C_{m-1}=0, C_{m} \neq 0$.

Under conditions A1-A3, the process $\{\varepsilon(t)=G(\xi(t)), t \in \mathbb{R}\}$, admits a Hermite series expansion in the Hilbert space $L_{2}(\Omega, \mathbb{F}, P)$ :

$$
\varepsilon(t)=G(\xi(t))=\sum_{k=m}^{\infty} \frac{C_{k}}{k !} H_{k}(\xi(t)) .
$$


We use the following modification of the LSE proposed by Walker (1973), see also Ivanov (1980, 2010). Consider a monotone non-decreasing system of open sets $S_{T} \subset S(\underline{\varphi}, \bar{\varphi}), T>T_{0}>0$, given by the condition that the true value of unknown parameter $\varphi$, belongs to $S_{T}$, and

$$
\lim _{T \rightarrow \infty} \inf _{1 \leq j<k \leq N,} T\left(\varphi_{k}-\varphi_{j}\right)=+\infty, \quad \lim _{T \rightarrow \infty} \inf _{\varphi \in S_{T}} T \varphi_{1}=+\infty
$$

where

$$
S(\underline{\varphi}, \bar{\varphi})=\left\{0 \leq \underline{\varphi}<\varphi_{1}<\cdots<\varphi_{N}<\bar{\varphi}<\infty\right\} .
$$

Remark 3 Assumption (10) allows to distinguish the parameters $\varphi_{k}, k=1, \ldots, N$, and prove the consistency of the LSE (see Theorem 1 below).

The LSE $\widehat{\theta}_{T}$ in the Walker sense of unknown parameter $\theta=\left(A_{1}, B_{1}, \varphi_{1}, \ldots, A_{N}, B_{N}, \varphi_{N}\right)$ in the model (11) with nonlinear regression function (3) is said to be any random vector $\hat{\theta}_{T} \in \Theta^{c}$ having the property:

$$
Q_{T}\left(\hat{\theta}_{T}\right)=\inf _{\tau \in \Theta^{c}} Q_{T}(\tau),
$$

where $Q_{T}(\tau)$ is defined in (2), and $\Theta \subset \mathbb{R}^{3 N}$ is such that $A_{k} \in \mathbb{R}, B_{k} \in \mathbb{R}, k=1, \ldots, N$, and $\varphi \in S_{T}^{c}$, the closure in $\mathbb{R}^{N}$ of the set $S_{T}$.

Remark 4 The 2-nd condition (10) is satisfied if $\underline{\varphi}>0$. If $S_{T} \subset S(\underline{\varphi}, \bar{\varphi})$, the relations given in (10) are, for example, satisfied for a parametric set $S_{T}$, such that

$$
\inf _{1 \leq j<k \leq N,}\left(\varphi_{k}-\varphi_{j}\right)=T^{-1 / 2}, \quad \inf _{\varphi \in S_{T}} \varphi_{1}=T^{-1 / 2} .
$$

Theorem 1 Under conditions $\boldsymbol{A} 1$ and $\boldsymbol{A} 2$, the LSE in the Walker sense

$$
\hat{\theta}_{T}=\left(\hat{A}_{1 T}, \hat{B}_{1 T}, \hat{\varphi}_{1 T}, \ldots, \hat{A}_{N T}, \hat{B}_{N T}, \hat{\varphi}_{N T}\right)
$$

of the unknown parameter $\theta=\left(A_{1}, B_{1}, \varphi_{1}, \ldots, A_{N}, B_{N}, \varphi_{N}\right)$ of the regression function (3) is weakly consistent as $T \rightarrow \infty$, that is,

$$
\hat{A}_{k_{T}} \stackrel{P}{\longrightarrow} A_{k}, \hat{B}_{k_{T}} \stackrel{P}{\longrightarrow} B_{k}, T\left(\hat{\varphi}_{k_{T}}-\varphi_{k}\right) \stackrel{P}{\longrightarrow} 0, \quad k=1, \ldots, N,
$$

where $\stackrel{P}{\longrightarrow}$ stands for the convergence in probability.

The proof of the Theorem 1 is based on the diagram technique. Let us first introduce the main elements involved in the definition of a diagram. Specifically, a graph $\Gamma=\Gamma\left(l_{1}, \ldots, l_{p}\right)$ with $l_{1}+\cdots+l_{p}$ vertices is called a diagram of order $\left(l_{1}, \ldots, l_{p}\right)$ if:

a) the set of vertices $V$ of the graph $\Gamma$ is of the form $V=\cup_{j=1}^{p} W_{j}$, where $W_{j}=\left\{(j, l), l=1, \ldots, l_{j}\right\}$ is the $j$ the level of the graph $\Gamma, 1 \leq j \leq p$ (if $l_{j}=0$, assume that $W_{j}=\emptyset$ );

b) each vertex is of degree 1 ;

c) if $\left(\left(j_{1}, l_{1}\right),\left(j_{2}, l_{2}\right)\right) \in \Gamma$ then $j_{1} \neq j_{2}$, that is, the edges of the graph $\Gamma$ may connect only different levels.

Let $L=L\left(l_{1}, \ldots, l_{p}\right)$ be a set of diagrams $\Gamma$ of order $\left(l_{1}, \ldots, l_{p}\right)$. Denote by $R(\Gamma)$ the set of edges of a graph $\Gamma \in L$. For the edge $\varpi=\left(\left(j_{1}, l_{1}\right),\left(j_{2}, l_{2}\right)\right) \in R(\Gamma), j_{1}<j_{2}$, we set $d_{1}(\varpi)=j_{1}, d_{2}(\varpi)=j_{2}$. We call a diagram $\Gamma$ regular if its levels can be split into pairs in such a manner that no edge connects the levels belonging to different pairs. We denote by $L^{*}$ the set of regular diagrams $L^{*} \subseteq L\left(l_{1}, \ldots, l_{p}\right)$, If $p$ is odd, then $L^{*}=\emptyset$. The following lemma provides the so called Diagram Formula (see Lemma 3.2, or Doukhan, Oppenheim and Taqqu (2003), p.74 or Pecatti and Taqqu (2010)).

Lemma 1 Let $\left(\zeta_{1}, \ldots, \zeta_{p}\right), p \geq 2$, be a Gaussian vector with $E \zeta_{j}=0, E \zeta_{j}^{2}=1, E \zeta_{i} \zeta_{j}=$ $B(i, j), i, j=1, \ldots, p$, and let $H_{l_{1}}(u), \ldots, H_{l_{p}}(u)$ be the Hermite polynomials. Then,

$$
E\left\{\prod_{j=1}^{p} H_{l_{j}}\left(\zeta_{j}\right)\right\}=\sum_{\Gamma \in L} I_{\Gamma},
$$

where $I_{\Gamma}=\prod_{\varpi \in R(\Gamma)} B\left(d_{1}(\varpi), d_{2}(\varpi)\right)$. 
As the special case for $p=2$ we have the following

$$
E H_{k}(\zeta(t)) H_{l}(\zeta(s))=k ! \delta_{k}^{l} B^{k}(t-s)
$$

where $\delta_{k}^{l}$ is the Kronecker delta.

Lemma 2 Suppose conditions $\boldsymbol{A} 1$ and $\boldsymbol{A} 2$ are fulfilled. Then

$$
\lim _{T \rightarrow \infty} E \eta^{2}(T)=0
$$

where $\eta(T)=\sup _{\lambda \in \mathbb{R}} \frac{1}{T}\left|\int_{0}^{T} e^{-i \lambda t} \varepsilon(t) d t\right|$.

Proof. Some ideas from Ivanov (2010) are used in the development of the proof of this lemma. First, from

$$
\left|\int_{0}^{T} e^{-i \lambda t} \varepsilon(t) d t\right|^{2}=\int_{-T}^{T} e^{-i \lambda u} \int_{0}^{T-|u|} \varepsilon(t+|u|) \varepsilon(t) d t d u=2 \int_{0}^{T} \cos \lambda u \int_{0}^{T-u} \varepsilon(t+u) \varepsilon(t) d t d u,
$$

we obtain

$$
\begin{aligned}
E \eta^{2}(T) & \leq \frac{2}{T^{2}} \int_{0}^{T} E\left|\int_{0}^{T-u} \varepsilon(t+u) \varepsilon(t) d t\right| d u \\
\leq & \sum_{i, j=1}^{2} \frac{2}{T^{2}} \int_{0}^{T} E\left|\int_{0}^{T-u} G_{i}(\xi(t+u)) G_{j}(\xi(t)) d t\right| d u=\sum_{i, j=1}^{2} I_{i, j}(T)
\end{aligned}
$$

with $G(x)=G_{1}(x)+G_{2}(x)$, where $G_{1}(x)=\sum_{k=1}^{M} \frac{C_{k}}{k !} H_{k}(x)$, and $G_{2}(x)=\sum_{k=M+1}^{\infty} \frac{C_{k}}{k !} H_{k}(x)$.

Let us now compute upper bounds for $I_{i, j}(T), i, j=1,2$. In particular, from Cauchy-Schwarz inequality:

$$
I_{12}(T) \leq\left(\frac{2}{T^{2}} \int_{0}^{T}(T-u) d u\right)\left(E G _ { 1 } ^ { 2 } ( \xi ( 0 ) ) ^ { 1 / 2 } \left(E G_{2}^{2}(\xi(0))^{1 / 2}<\left(E G_{1}^{2}(\xi(0))^{1 / 2} \epsilon,\right.\right.\right.
$$

since from (8),$E G_{2}^{2}(\xi(0))=\sum_{k=M+1}^{\infty} \frac{C_{k}^{2}}{k !}$ is the tail of a convergent series, and similarly, $I_{21}(T)<$ $\left(E G_{1}^{2}(\xi(0))^{1 / 2} \epsilon\right.$, and $I_{22}(T)<\epsilon^{2}$, for any $\epsilon>0$.

Note that

$$
I_{11}(T)=\frac{2}{T^{2}} \int_{0}^{T} E\left|\int_{0}^{T-u} G_{1}(\xi(t+u)) G_{1}(\xi(t)) d t\right| d u \leq \frac{2}{T^{2}} \int_{0}^{T} \psi^{1 / 2}(u) d u,
$$

where

$$
\begin{aligned}
\psi(u) & =\int_{0}^{T-u} \int_{0}^{T-u} E G_{1}(\xi(t+u)) G_{1}(\xi(s+u)) G_{1}(\xi(t)) G_{1}(\xi(s)) d t d s \\
& =\sum_{l_{1}, l_{2}, l_{3}, l_{4}=1}^{M}\left(\prod_{j=1}^{4} \frac{C_{l_{j}}}{l_{j} !}\right) \int_{0}^{T-u} \int_{0}^{T-u} E\left(\prod_{j=1}^{4} H_{l_{j}}\left(\xi_{j}\right)\right) d t d s,
\end{aligned}
$$

with $\left(\xi_{1}, \xi_{2}, \xi_{3}, \xi_{4}\right)=(\xi(t+u), \xi(s+u), \xi(t), \xi(s))$.

Applying diagram formula (12) with $p=4$, for $\Gamma \in L^{*}\left(l_{1}, l_{2}, l_{3}, l_{4}\right)$, we have different splitting of the levels $(1,2,3,4)$ into pairs:

(i) $\quad(1,2) \quad(3,4) \quad(i i) \quad(1,3) \quad(2,4) \quad($ iii $) \quad(1,4) \quad(2,3)$.

Let us denote the cardinality of the levels of the first pairs in (i), (ii) and (iii) as $r(1$ ), and the cardinality of the levels of the second pairs in (i), (ii) and (iii) as $r(2) ; r(1)$ and $r(2)$ are the orders of Hermite polynomials in the left-hand side of (12). 
For the product

$$
\prod_{\varpi \in R(\Gamma)} B\left(d_{1}(\varpi), d_{2}(\varpi)\right)
$$

in (12), we obtain the following estimates: In case (i), product (17) is bounded by $B^{r(1)+r(2)}(t-s)$, while, for the cases (ii) and (iii), the expression (17) is bounded by $B^{r(1)+r(2)}(u)$, and $B^{r(1)}(t-s+$ $u) B^{r(2)}(t-s-u)$, respectively.

From (12), (15), (16) and (17), we have

$$
I_{11}(T) \leq 2 \sum_{l_{1}, l_{2}, l_{3}, l_{4}=1}^{M}\left(\prod_{j=1}^{4} \frac{C_{l_{j}}}{l_{j} !}\right) \sum_{\Gamma \in L} \frac{1}{T^{2}} \int_{0}^{T}\left[\int_{0}^{T-u} \int_{0}^{T-u} \prod_{\varpi \in R(\Gamma)}\left|B\left(d_{1}(\varpi), d_{2}(\varpi)\right)\right| d t d s\right]^{1 / 2} d u .
$$

Since $r(1), r(2) \geq 1$, we need to estimate for the regular diagram in (18) the following integrals:

$$
\frac{1}{T^{2}} \int_{0}^{T} \gamma_{i}^{1 / 2}(u) d u, i=1,2,3
$$

where

$$
\begin{aligned}
& \gamma_{1}(u)=\int_{0}^{T-u} \int_{0}^{T-u} B^{2}(t-s) d t d s, \quad \gamma_{2}(u)=(T-u)^{2} B^{2}(u), \\
& \gamma_{3}(u)=\int_{0}^{T-u} \int_{0}^{T-u}|B(t-s+u) B(t-s-u)| d t d s .
\end{aligned}
$$

Note that

$$
|B(t)|=\left|\sum_{j=0}^{\kappa} D_{j} B_{\alpha_{j}, \varkappa_{j}}(t)\right| \leq\left(1+t^{2}\right)^{-\alpha / 2}=B_{0}(t)
$$

Consider first the case $\alpha=\min \left(\alpha_{0}, \ldots, \alpha_{r}\right) \in(0,1)$. Introduce the function $L(t)=\left[\frac{t^{2}}{1+t^{2}}\right]^{\alpha / 2}$ being monotonically nondecreasing slowly varying at infinity function (s.v.f), see Seneta (1976). Then, $B_{0}(t)=\frac{L(t)}{|t|^{\alpha}}$.

Denote by $b_{u}^{0}(t-s)=B_{0}(t-s-u) B_{0}(t-s+u)$, with

$$
B_{0}(t)=\frac{L(t)}{|t|^{\alpha}} .
$$

Using the change of variables $\left(t \mapsto \frac{t}{T}, s \rightarrow \frac{s}{T}, t-s \rightarrow t\right)$, we obtain

$$
\begin{aligned}
\gamma_{3}(u) & \leq T^{2} \int_{0}^{1-\frac{u}{T}} \int_{0}^{1-\frac{u}{T}}\left|b_{u}^{0}(T(t-s))\right| d t d s=T^{2}\left(1-\frac{u}{T}\right) \int_{-1+\frac{u}{T}}^{1+\frac{u}{T}}\left(1-\frac{|t|}{1-\frac{u}{T}}\right)\left|b_{u}^{0}(T t)\right| d t \\
& \leq T^{2}\left(1-\frac{u}{T}\right) \int_{-1}^{1}\left|b_{u}^{0}(T t)\right| d t \leq T^{2}\left(1-\frac{u}{T}\right)\left[\int_{0}^{1}\left|B_{0}(T t+u)\right| d t+\int_{-1}^{0}\left|B_{0}(T t-u)\right| d t\right] .
\end{aligned}
$$

Since

$$
\int_{-1}^{0} B_{0}(T t-u) d t=\int_{0}^{1} B_{0}(T t+u) d t
$$

we rewrite

$$
\gamma_{3}(u) \leq 2 T^{2}\left(1-\frac{u}{T}\right) \int_{0}^{1}\left|B_{0}(T t+u)\right| d t
$$

From $\mathbf{A} 1$ and the monotonicity of the function $L(t)$, for any $\epsilon>0$, and, for $T$ sufficiently large, the following inequalities hold:

$$
B_{0}(T t+u)=\frac{L(T t+u)}{|T t+u|^{\alpha}} \leq \frac{L(2 T)}{(T t)^{\alpha}}<\frac{1+\epsilon}{t^{\alpha}} \frac{L(T)}{T^{\alpha}}=\frac{1+\epsilon}{t^{\alpha}} B_{0}(T)
$$


and, hence,

$$
\frac{1}{T^{2}} \int_{0}^{T} \gamma_{3}^{1 / 2}(u) d u \leq \frac{2 \sqrt{2}}{3}\left(\frac{1+\epsilon}{1-\alpha}\right)^{1 / 2} B_{0}^{1 / 2}(T)
$$

Similarly, we have $\gamma_{1}(u) \leq 2 T^{2}\left(1-\frac{u}{T}\right) \int_{0}^{1} B_{0}^{2}(T t) d t \leq 2 T^{2}\left(1-\frac{u}{T}\right) \int_{0}^{1} B_{0}(T t) d t$. Thus,

$$
\frac{1}{T^{2}} \int_{0}^{T} \gamma_{1}^{1 / 2}(u) d u \leq \frac{2 \sqrt{2}}{3 \sqrt{1-\alpha}} B_{0}^{1 / 2}(T)
$$

On the other hand,

$$
\begin{aligned}
\frac{1}{T^{2}} \int_{0}^{T} \gamma_{2}^{1 / 2}(u) d u & \leq \frac{1}{T^{2}} \int_{0}^{T}(T-u)|B(u)| d u \\
& \leq \int_{0}^{1}(1-u) B_{0}(T u) d u \leq \frac{1}{(1-\alpha)(2-\alpha)} B_{0}(T)
\end{aligned}
$$

Thus, all the terms in (16), corresponding to the regular diagrams, tend to zero as $T \rightarrow \infty$.

Let us now consider the non-regular diagrams in (16). Fix $\Gamma \in L \backslash L^{*}$. In the product

$$
\prod_{\varpi \in R(\Gamma)} B\left(d_{1}(\varpi), d_{2}(\varpi)\right)
$$

there is a multiplier $B(t-s)$ (which means that an edge between levels 1 and 2 or 2 and 4 exists), or there is a multiplier $B(u)$ (which means that an edge between the levels 1 and 3 or 2 and 4 exists). If the diagram $\Gamma$ has no edges with such properties, then, level 1 will be connected to level 4 , and level 2 will be connected to level 3, which is true for regular diagram only. Thus, expression (18) is given in terms of either $B(t-s)$ or $B(u)$, and similarly to (21) and (22) one can obtain

$$
\frac{1}{T^{2}} \int_{0}^{T}\left[\int_{0}^{T-u} \int_{0}^{T-u}|B(t-s)| d t d s\right]^{1 / 2} d u \leq \frac{2 \sqrt{2}}{3(1-\alpha)} B_{0}^{1 / 2}(T)
$$

and

$$
\frac{1}{T^{2}} \int_{0}^{T}(T-u)|B(u)|^{1 / 2} d u \leq \frac{4}{(2-\alpha)(4-\alpha)} B_{0}^{1 / 2}(T) .
$$

From (20)-(25), expression (18) tends to zero when $T \longrightarrow \infty$, and hence, the statement of Lemma 2 follows, for $\alpha<1$. The case $\alpha>1$ is almost obvious because of the integrability of the function $B_{0}(t)$. If $\alpha=1$, integrals of $B_{0}(t)$ are of logarithmic order in $T$ and the statement of Lemma 2 is also true.

The proof of Theorem 1 is now derived.

\section{Proof.}

Denote

$$
z_{k_{T}}=\frac{\sin \left[T\left(\hat{\varphi}_{k_{T}}-\varphi_{k}\right)\right]}{T\left(\hat{\varphi}_{k_{T}}-\varphi_{k}\right)}, \quad y_{k_{T}}=\frac{1-\cos \left[T\left(\hat{\varphi}_{k_{T}}-\varphi_{k}\right)\right]}{T\left(\hat{\varphi}_{k_{T}}-\varphi_{k}\right)} .
$$

We shall show that for $k=1,2, \ldots, N$,

$$
\begin{aligned}
& \hat{A}_{k_{T}}=A_{k} z_{k_{T}}-B_{k} y_{k_{T}}+o_{P}(1), \\
& \hat{B}_{k_{T}}=A_{k} y_{k_{T}}+B_{k} z_{k_{T}}+o_{P}(1),
\end{aligned}
$$

where $o_{P}(1)$ means (different) stochastic processes tending to zero in probability as $T \longrightarrow \infty$.

Taking derivatives of the functional $Q_{T}(\theta)$ with respect to $A_{k}, B_{k}, k=1,2, \ldots, N$, we obtain the following system of linear equations in terms of the LSE $\hat{A}_{k_{T}}, \hat{B}_{k_{T}}, k=1,2, \ldots, N$ :

$$
\left\{\begin{array}{l}
\sum_{k=1}^{N} a_{k j}^{(1)}(T) \hat{A}_{k_{T}}+\sum_{k=1}^{N} b_{k j}^{(1)}(T) \hat{B}_{k_{T}}=c_{j}^{(1)}(T), \\
\sum_{k=1}^{N} a_{k j}^{(2)}(T) \hat{A}_{k_{T}}+\sum_{k=1}^{N} b_{k j}^{(2)}(T) \hat{B}_{k_{T}}=c_{j}^{(2)}(T)
\end{array}, j=1, \ldots, N,\right.
$$


where, denoting

$$
<u(t), v(t)>=\frac{1}{T} \int_{0}^{T} u(t) v(t) d t
$$

for $k, j=1, \ldots, N$,

$$
\begin{array}{cc}
a_{k j}^{(1)}(T)=\left\langle\cos \left(\hat{\varphi}_{k_{T}} t\right), \cos \left(\hat{\varphi}_{j_{T}} t\right)\right\rangle, & a_{k j}^{(2)}(T)=\left\langle\cos \left(\hat{\varphi}_{k_{T}} t\right), \sin \left(\hat{\varphi}_{j_{T}} t\right)\right\rangle, \\
b_{k j}^{(1)}(T)=\left\langle\sin \left(\hat{\varphi}_{k_{T}} t\right), \cos \left(\hat{\varphi}_{j_{T}} t\right)\right\rangle, & b_{k j}^{(2)}(T)=\left\langle\sin \left(\hat{\varphi}_{k_{T}} t\right), \sin \left(\hat{\varphi}_{j_{T}} t\right)\right\rangle, \\
c_{j}^{(1)}(T)=\left\langle x(t), \cos \left(\hat{\varphi}_{j_{T}} t\right)\right\rangle, & c_{j}^{(2)}(T)=\left\langle x(t), \sin \left(\hat{\varphi}_{j_{T}} t\right)\right\rangle .
\end{array}
$$

From (10), we have the following, for $k, j=1, \ldots, N$,

$$
\begin{gathered}
a_{k j}^{(1)}(T)=a_{k j}^{(2)}(T)=o(1), \quad k \neq j, \quad a_{k k}^{(1)}(T)=\frac{1}{2}+o(1), \\
b_{k j}^{(1)}(T)=b_{k j}^{(2)}(T)=o(1), \quad k \neq j, \quad b_{k k}^{(2)}(T)=\frac{1}{2}+o(1),
\end{gathered}
$$

where $o(1)$ means (different) stochastic processes tending to zero almost surely, as $T \longrightarrow \infty$.

Then, one can continue as follows:

$$
c_{j}^{(1)}(T)=\left\langle\varepsilon(t), \cos \left(\widehat{\varphi}_{j_{T}} t\right)\right\rangle+\left\langle g(t, \theta), \cos \left(\widehat{\varphi}_{j_{T}} t\right)\right\rangle=d_{j}^{(1)}(T)+d_{j}^{(2)}(T)
$$

where $d_{j}^{(1)}(T)=o_{P}(1)$ by Lemma 2, and

$$
\begin{aligned}
d_{j}^{(2)}(T) & =A_{j}^{0}\left\langle\cos \left(\varphi_{j} t\right), \cos \left(\hat{\varphi}_{j_{T}} t\right)\right\rangle+B_{j}^{0}\left\langle\sin \left(\varphi_{j} t\right), \cos \left(\hat{\varphi}_{j_{T}} t\right)\right\rangle+o_{P}(1), \\
& =\frac{1}{2}\left[A_{j} z_{j T}-B_{j} y_{j T}\right]+o_{P}(1),
\end{aligned}
$$

or

$$
c_{j}^{(1)}(T)=\frac{1}{2}\left[A_{j} z_{j_{T}}-B_{j} y_{j_{T}}\right]+o_{P}(1), j=1, \ldots, N,
$$

and similarly

$$
c_{j}^{(2)}(T)=\frac{1}{2}\left[A_{j} y_{j_{T}}+B_{j} z_{j_{T}}\right]+o_{P}(1), j=1, \ldots, N,
$$

where $o_{P}(1)$ are processes tending to zero in probability as $T \rightarrow \infty$.

Since $\left|z_{j_{T}}\right|,\left|y_{j_{T}}\right| \leq 1$, we obtain, from (26),

$$
\left|\hat{A}_{k_{T}}\right|\left|\hat{B}_{k_{T}}\right| \leq\left|A_{k}\right|+\left|B_{k}\right|+o_{P}(1), \quad k=1, \ldots, N .
$$

Let $\Delta g\left(t ; \theta_{1}, \theta_{2}\right)=g\left(t, \theta_{1}\right)-g\left(t, \theta_{2}\right)$, and $K_{T}\left(\theta_{1}, \theta_{2}\right)=\left\langle\Delta g\left(t ; \theta_{1}, \theta_{2}\right), \Delta g\left(t ; \theta_{1}, \theta_{2}\right)\right\rangle$.

By definition of LSE

$$
Q_{T}\left(\hat{\theta}_{T}\right) \leq Q_{T}(\theta) .
$$

On the other hand,

$$
Q_{T}\left(\hat{\theta}_{T}\right)-Q_{T}\left(\theta_{0}\right)=K_{T}\left(\hat{\theta}_{T}, \theta_{0}\right)+2\left\langle\varepsilon(t), \Delta g\left(t ; \theta_{1}, \hat{\theta}_{T}\right)\right\rangle,
$$

and by Lemma 2 and (30)-(32), we have

$$
\left\langle\varepsilon(t), \Delta g\left(t ; \theta_{1}, \hat{\theta}_{T}\right)\right\rangle=o_{P}(1) .
$$

From (33), (34) and (35), it follows that

$$
K_{T}\left(\hat{\theta}_{T}, \theta\right)=o_{P}(1) .
$$


Consider $g_{k_{T}}(t)=\hat{A}_{k_{T}} \cos \left(\hat{\varphi}_{k_{T}} t\right)+\hat{B}_{k_{T}} \sin \left(\hat{\varphi}_{j_{T}} t\right)-A_{k} \cos \left(\varphi_{k} t\right)-B_{k} \sin \left(\varphi_{k} t\right)$. Observe that

$$
K_{T}\left(\hat{\theta}_{T}, \theta\right)=\sum_{k=1}^{N}\left\langle g_{k_{T}}(t), g_{k_{T}}(t)\right\rangle+2 \sum_{k<j}^{N}\left\langle g_{k_{T}}(t), g_{j_{T}}(t)\right\rangle, \quad k \neq j .
$$

In a similar way as before, for $k=1, \ldots, N$,

$$
\begin{aligned}
\left\langle g_{k_{T}}(t), g_{j_{T}}(t)\right\rangle & =o_{P}(1), \quad k \neq j, \\
\left\langle g_{k_{T}}(t), g_{k_{T}}(t)\right\rangle= & \frac{1}{2}\left[\hat{A}_{k_{T}}^{2}+\hat{B}_{k_{T}}^{2}+\left(A_{k}\right)^{2}+\left(B_{k}\right)^{2}\right] \\
& -\left(\hat{A}_{k_{T}} A_{k}+\hat{B}_{k_{T}} B_{k}\right) z_{k_{T}}+\left(\hat{A}_{k_{T}} B_{k}+\hat{B}_{k_{T}} A_{k}\right) y_{k_{T}}+o_{P}(1) .
\end{aligned}
$$

From (26)-(34), we get

$$
\begin{aligned}
K_{T}\left(\hat{\theta}_{T}, \theta\right) & =\frac{1}{2} \sum_{k=1}^{N}\left(\left(A_{k}\right)^{2}+\left(B_{k}\right)^{2}\right)\left(1-z_{k_{T}}^{2}-y_{k_{T}}^{2}\right)+o_{P}(1) \\
& =\frac{1}{2} \sum_{k=1}^{N}\left(\left(A_{k}\right)^{2}+\left(B_{k}\right)^{2}\right)\left(1-\left(\frac{\sin \left(\frac{1}{2} T\left(\hat{\varphi}_{k_{T}}-\varphi_{k}\right)\right)}{\frac{1}{2} T\left(\hat{\varphi}_{k_{T}}-\varphi_{k}\right)}\right)^{2}\right)+o_{P}(1) .
\end{aligned}
$$

Additionally, from (36), we have

$$
1-\left(\frac{\sin \left(\frac{1}{2} T\left(\hat{\varphi}_{k_{T}}-\varphi_{k}\right)\right)}{\frac{1}{2} T\left(\hat{\varphi}_{k_{T}}-\varphi_{k}\right)}\right)=o_{P}(1), \quad k=1, \ldots, N
$$

Since $\frac{\sin x}{x}, x \geq 0$, is decreasing around zero, then, for $\epsilon \in(0,1)$, thanks to (39),

$$
P\left\{\frac{1}{2} T\left|\hat{\varphi}_{k_{T}}-\varphi\right|>\epsilon\right\} \leq P\left\{\left(1-\frac{\sin \left(\frac{1}{2} T\left(\hat{\varphi}_{k_{T}}-\varphi\right)\right)}{\frac{1}{2} T\left(\hat{\varphi}_{k_{T}}-\varphi\right)}\right)>1-\frac{\sin \epsilon}{\epsilon}\right\} \longrightarrow 0, \quad T \longrightarrow \infty
$$

or

$$
T\left(\hat{\varphi}_{k_{T}}-\varphi\right)=o_{P}(1), \quad T \longrightarrow \infty .
$$

Observe that $z_{k_{T}}=\frac{\sin \left(\frac{1}{2} T\left(\hat{\varphi}_{k_{T}}-\varphi\right)\right)}{\frac{1}{2} T\left(\hat{\varphi}_{k_{T}}-\varphi\right)} \cos \left(\frac{1}{2} T\left(\hat{\varphi}_{k_{T}}-\varphi_{k}\right)\right)$. Since $1-\cos x<\frac{x^{2}}{2}, x>0$, using (40), we see that, for any $\epsilon>0$,

$$
P\left\{1-\cos \left(\frac{1}{2} T\left(\hat{\varphi}_{k_{T}}-\varphi\right)\right)>\epsilon\right\} \leq P\left\{T\left|\hat{\varphi}_{k_{T}}-\varphi\right|>2 \sqrt{2 \epsilon}\right\} \longrightarrow 0, \quad T \longrightarrow \infty .
$$

From (39) and (41), we get that $z_{k_{T}}=1+o_{P}(1), k=1, \ldots, N$. Moreover, from (40), we obtain $\sin \left(\frac{1}{2} T\left(\hat{\varphi}_{k_{T}}-\varphi\right)\right)=o_{P}(1)$, and thus, $y_{k_{T}}=o_{P}(1), k=1, \ldots, N$. Finally, from (26), we then have

$$
\hat{A}_{k_{T}} \stackrel{P}{\longrightarrow} A_{k}, \hat{B}_{k_{T}} \stackrel{P}{\longrightarrow} B_{k}, \quad k=1, \ldots, N, \quad T \longrightarrow \infty .
$$

\section{Linearization and asymptotic uniqueness}

This section reviews and clarifies a number of results, on non-linear regression, in particular, from Ivanov and Leonenko $(2004,2008,2009)$. Consider the general non-linear regression model (1) with the noise process satisfying condition A2. Let $\hat{\theta}_{T}$ be the LSE of an unknown parameter $\theta$, that is, a random vector $\hat{\theta}_{T} \in \Theta^{c}$ having the property (11). The following assumption is considered.

B1. Suppose that $g(t, \tau)$ is twice differentiable with respect to $\tau \in \Theta^{c}$. 
Under B1, we then get

$$
\begin{gathered}
g_{i}(t, \tau)=\frac{\partial}{\partial \tau_{i}} g(t, \tau), \quad g_{i l}(t, \tau)=\frac{\partial^{2}}{\partial \tau_{i} \partial \tau_{l}} g(t, \tau), \quad i, l=1, \ldots, q, \\
d_{T}(\tau)=\operatorname{diag}\left(d_{i T}(\tau)\right)_{i=1}^{q}, \quad \tau \in \Theta^{c}, \quad d_{i T}^{2}(\theta)=\int_{0}^{T} g_{i}^{2}(t, \theta) d t \quad i=1, \ldots, q .
\end{gathered}
$$

Additionally, let us assume:

B2 The following positive limits exist $\underline{\lim }_{T \rightarrow \infty} T^{-1} d_{i T}^{2}(\theta)>0$, for $i=1, \ldots, q$.

Note that the limits in (42) can be, in particular, not finite. Let also

$$
d_{i l, T}^{2}(\tau)=\int_{0}^{T} g_{i l}^{2}(t, \tau) d t, \quad \tau \in \Theta^{c}, \quad i, l=1, \ldots, q .
$$

Consider now the normalized LSE

$$
\hat{u}_{T}=d_{T}(\theta)\left(\hat{\theta}_{T}-\theta\right),
$$

and the notation: $g\left(t, \theta+d_{T}^{-1}(\theta) u\right)=h(t, u), g_{i}\left(t, \theta+d_{T}^{-1}(\theta) u\right)=h_{i}(t, u), i=1, \ldots, q, g_{i l}(t, \theta+$ $\left.d_{T}^{-1}(\theta) u\right)=h_{i l}(t, u)$, for $i, l=1, \ldots, q$, as well as $V(R)=\left\{u \in \mathbb{R}^{q}:\|u\|<R\right\}$ for the ball of radius $R$. Here, the following change of variables is performed: $u=d_{T}(\theta)(\tau-\theta)$. The letter $k$ will be used for denoting positive constants. The following assumptions are formulated, for $R \geq 0, \theta \in \Theta$, and $T>T_{0}(R)$ sufficiently large:

$$
\begin{aligned}
& \text { B3 } \sup _{t \in[0, T]} \sup _{u \in V^{c}(R)} \frac{\left|h_{i}(t, u)\right|}{d_{i T}(\theta)} \leq k^{i}(R) T^{-1 / 2}, \\
& \sup _{t \in[0, T]} \sup _{u \in V^{c}(R)} \frac{\left|h_{i l}(t, u)\right|}{d_{i l, T}(\theta)} \leq k^{i l}(R) T^{-1 / 2}, \sup _{t \in[0, T]} \sup _{\theta \in V^{c}(R)} \frac{d_{i l, T}(\theta)}{d_{i T}(\theta) d_{l T}(\theta)} \leq \tilde{k}^{i l}(R) T^{-1 / 2}, \quad i, l=1, \ldots, q .
\end{aligned}
$$

We will use the notation: $H\left(t ; u_{1}, u_{2}\right)=h\left(t, u_{1}\right)-h\left(t, u_{2}\right), H_{i}\left(t ; u_{1}, u_{2}\right)=h_{i}\left(t, u_{1}\right)-h_{i}\left(t, u_{2}\right)$, for $i=1, \ldots, q$. Introduce also the vectors $\psi_{T}(u)=\left(\psi_{T}^{i}(u)\right)_{i=1}^{q}$, with

$$
\psi_{T}^{i}(u)=\int_{0}^{T} \varepsilon(t) \frac{h_{i}(t, u)}{d_{i T}(\theta)} d t+\int_{0}^{T} H(t ; 0, u) \frac{h_{i}(t, u)}{d_{i T}(\theta)} d t,
$$

and $L_{T}(u)=\left(L_{T}^{i}(u)\right)_{i=1}^{q}$, with

$$
L_{T}^{i}(u)=\int_{0}^{T}\left(\varepsilon(t)-\sum_{l=1}^{q} \frac{g_{l}(t, \theta)}{d_{l T}(\theta)} u_{l}\right) \frac{g_{i}(t, \theta)}{d_{i T}(\theta)} d t, \quad i=1, \ldots, q .
$$

The vectors (46) and (47) are defined for $u \in U_{T}^{c}(\theta)$, where $U_{T}(\theta)=d_{T}(\theta)(\Theta-\theta)$. Note that, under our assumptions, for any $R>0, V^{c}(R) \subset U_{T}(\theta)$, for $T>T_{0}(R)$.

The normalized LSE $\hat{u}_{T}$ satisfies the system of normal equations:

$$
\psi_{T}(u)=0,
$$

while the vector $L_{T}(\theta)$ corresponds to the auxiliary linear regression model:

$$
Z(t)=\sum_{i=1}^{q} g_{i}(t, \theta) \beta_{i}+\varepsilon(t), t \in[0, T] .
$$

The system of normal equations for the linear regression model (49)

$$
L_{T}(\theta)=0,
$$

determines the normed LSE $\tilde{u}_{T}$ of the parameter $\beta$, if

$$
\tilde{u}_{T}=d_{T}(\theta)\left(\tilde{\beta}_{T}-\beta\right),
$$

where $\tilde{\beta}_{T}$ is the ordinary LSE of the parameter $\beta$ in the model (49). 
Theorem 2 Under the assumptions $\boldsymbol{A} \mathbf{1 - A} 3$ and $\boldsymbol{B} 1-B 3$, for any $R>0, r>0$

$$
P\left\{\sup _{u \in V^{c}(R)}\left\|\psi_{T}(u)-L_{T}(u)\right\|>r\right\} \longrightarrow 0, T \longrightarrow \infty .
$$

The proof of Theorem 2 is given in Appendix 1.

In this section, we show that the LSE $\hat{\theta}_{T}$ in certain sense is the asymptotically unique solution of the system of normal equations (48) as $T \longrightarrow \infty$. Let us first consider $J_{T}(\theta)=\left(J_{i l, T}(\theta)\right)_{i, l=1}^{q}$, where

$$
J_{i l, T}(\theta)=d_{i T}^{-1}(\theta) d_{l T}^{-1}(\theta) \int_{0}^{T} g_{i}(t, \theta) g_{l}(t, \theta) d t .
$$

Denote by $\lambda_{\min }(A)$ and $\lambda_{\max }(A)$ the respective minimal and maximal eigenvalues of a positive definite matrix $A$. Let us formulate the next condition:

B4 For some $\lambda_{*}>0$ and $T>T_{0}$,

$$
\lambda_{\min }\left(J_{T}(\theta)\right) \geq \lambda_{*} .
$$

Consider now the normed LSE

$$
\hat{w}_{T}=T^{-1 / 2} d_{T}(\theta)\left(\hat{\theta}_{T}-\theta\right),
$$

where the change of variables $w=T^{-1 / 2} d_{T}(\theta)(\tau-\theta)$ is applied into the regression function and its derivatives. The following notation is established: For $i, l=1, \ldots q$,

$f(t, w)=g\left(t, \theta+T^{1 / 2} d_{T}^{-1}(\theta) w\right), f_{i}(t, w)=g_{i}\left(t, \theta+T^{1 / 2} d_{T}^{-1}(\theta) w\right), f_{i l}(t, w)=g_{i l}\left(t, \theta+T^{1 / 2} d_{T}^{-1}(\theta) w\right)$.

Additionally, we denote, for $i, l=1, \ldots q, F\left(t ; w_{1}, w_{2}\right)=f\left(t, w_{1}\right)-f\left(t, w_{2}\right)$,

$F_{i}\left(t ; w_{1}, w_{2}\right)=f_{i}\left(t, w_{1}\right)-f_{i}\left(t, w_{2}\right), F_{i l}\left(t ; w_{1}, w_{2}\right)=f_{i l}\left(t, w_{1}\right)-f_{i l}\left(t, w_{2}\right), \Phi_{i l, T}(w, 0)=\int_{0}^{T} F_{i l, T}^{2}(t ; w, 0) d t$.

Finally, the following assumption is considered in the derivation of Theorem 3 below:

B5 For some $\tau_{0}>0$, and for $i, l=1, \ldots q, \quad \sup _{t \in[0, T]} \sup _{w \in V^{c}\left(\tau_{0}\right)} \frac{\left|f_{i}(t, w)\right|}{d_{i T}(\theta)} \leq \tilde{k}^{i}\left(\tau_{0}\right) T^{-1 / 2}$,

$$
\sup _{t \in[0, T]} \sup _{w \in V^{c}\left(\tau_{0}\right)} \frac{\left|f_{i l}(t, w)\right|}{d_{i l, T}(\theta)} \leq \tilde{k}^{i l}\left(\tau_{0}\right) T^{-1 / 2}, \sup _{w \in V^{c}\left(\tau_{0}\right)} T d_{i T}^{-2}(\theta) d_{l T}^{-2}(\theta) \Phi_{i l, T}(w, 0)\|w\|^{-2} \leq \hat{k}_{i l}\left(\tau_{0}\right) .
$$

Consider the functional

$$
(2 T)^{-1} \int_{0}^{T}[x(t)-f(t, w)]^{2} d t=\frac{1}{2} Q_{T}\left(\theta+T^{1 / 2} d_{T}^{-1}(\theta) w\right),
$$

and the vector

$\mathbf{M}_{T}(w)=\left(M_{T}^{i}(w)\right)_{i=1}^{q}=\left(\frac{\partial}{\partial w_{i}}\left(\frac{1}{2} Q_{T}\left(\theta+T^{1 / 2} d_{T}^{-1}(\theta) w\right)\right)\right)_{i=1}^{q}=\left(T^{-1 / 2} \int_{0}^{T}[x(t)-f(t, w)] \frac{-f_{i}(t, w)}{d_{i T}(\theta)} d t\right)_{i=1}^{q}$.

Then, the normed LSE (54) satisfies the system of equations

$$
\mathbf{M}_{T}(w)=0 .
$$

C For any $r>0$,

$$
P\left\{\left\|\hat{w}_{T}\right\|>r\right\} \longrightarrow 0, \quad T \longrightarrow \infty .
$$

Note that if the normed LSE $\hat{w}_{T}$ is an unique solution of the system of equations (55), then, the LSE $\hat{u}_{T}$ is the unique solution of the system (48).

Theorem 3 Under conditions $\boldsymbol{A} \mathbf{1 - A 3 ,} \boldsymbol{B 1 - B 5}$ and $\boldsymbol{C}$, the normed LSE (54) is an unique solution of the system of equations (55) with probability tending to 1 , as $T \longrightarrow \infty$.

We place the proof of this theorem into Appendix 2.

Remark 5 The verification of the conditions B3 - B5 fulfilment for regression function (3) is not difficult and we omit it. 


\section{Central limit theorem}

This section is derivation of the convergence to the Gaussian distribution. This convergence result is obtained, under conditions, for the integral functional

$$
\zeta_{T}=d_{T}^{-1}(\theta) \int_{0}^{T} \nabla g(t, \theta) G(\xi(t)) d t,
$$

as $T \longrightarrow \infty$, where $g(t, \theta)$ is the general regression function and $\nabla g(t, \theta)=\left(g_{i}(t, \theta)\right)_{i=1}^{q}$ is its gradient. We introduce a family of a matrix-valued measures $\boldsymbol{\mu}_{T}(d \lambda)=\left(\mu_{T}^{j l}(d \lambda, \theta)\right)_{j, l=1}^{q}, T>0$, where $\mu_{T}^{j l}(\lambda, \theta)$ are given by (4). Using the notation (4)-(5) and (42) note that

$$
d_{j T}^{2}(\theta)=\frac{1}{2 \pi} \int_{\mathbb{R}}\left|g_{T}^{j}(\lambda, \theta)\right|^{2} d \lambda, \quad j=1, \ldots, q
$$

B6 The family of measures $\boldsymbol{\mu}_{T}(d \lambda)$ converges weakly to the measure $\boldsymbol{\mu}(d \lambda, \theta)=\left(\mu^{j l}(d \lambda, \theta)\right)_{j, l=1}^{q}$, as $T \longrightarrow \infty$.

Condition B6 means that the elements $\mu^{j l}(d \lambda, \theta)$ of the matrix $\boldsymbol{\mu}(d \lambda, \theta)$ are complex signed measures of bounded variation and the matrix $\boldsymbol{\mu}(\mathcal{A}, \theta)$ is positive semi-definite for any Borel set $\mathcal{A}$. The limiting measure $\boldsymbol{\mu}(d \lambda, \theta)$ is called the spectral measure of the regression function $g(t, \theta)$, see Grenander and Rosenblatt (1984), Holevo (1976), Ibragimov and Rozanov (1980), Ivanov and Leonenko (1989). Note that

$$
\begin{aligned}
\int_{\mathbb{R}} \boldsymbol{\mu}_{T}(d \lambda, \theta) & =\left(\int_{\mathbb{R}} \mu_{T}^{j l}(d \lambda, \theta)\right)_{j, l=1}^{q} \\
& =\left(d_{j T}^{-1}(\theta) d_{l T}^{-1}(\theta) \int_{0}^{T} g_{j}(t, \theta) g_{l}(t, \theta) d t\right)_{j, l=1}^{q}=\left(J_{j l, T}(\theta)\right)_{j, l=1}^{q}=J_{T}(\theta),
\end{aligned}
$$

and $J_{T}(\theta) \longrightarrow J(\theta)$, as $T \longrightarrow \infty$, for matrix $J(\theta)>0$, such that $\lambda_{\min }(J(\theta)) \geq \tilde{\lambda}_{*}>0$. We then have

$$
J(\theta)=\left(\int_{\mathbb{R}} \mu^{j l}(d \lambda, \theta)\right)_{j, l=1}^{q}=\int_{\mathbb{R}} \boldsymbol{\mu}(d \lambda, \theta) .
$$

Note also that

$$
\zeta_{T}=\left(\int_{0}^{T} G(\xi(t)) v_{i T}(t, \theta) d t\right)_{i=1}^{q}
$$

where

$$
v_{i T}(t, \theta)=g_{i}(t, \theta) d_{i T}^{-1}(t, \theta) .
$$

The weak convergence of the random vector

$$
\zeta_{T}=\left(\zeta_{1 T}, \ldots, \zeta_{q T}\right) \Rightarrow\left(\zeta_{1}, \ldots, \zeta_{q}\right)=\zeta,
$$

is equivalent to convergence of the characteristic functions: for any $z \in \mathbb{R}^{q}$,

$$
E \exp \left\{i u\left\langle\zeta_{T}, z\right\rangle\right\} \underset{T \longrightarrow \infty}{\longrightarrow} E \exp \{i u\langle\zeta, z\rangle\}, u \in \mathbb{R}^{1} .
$$

Thus, the convergence in (57) will follow from (58).

Under the condition A3, consider expansion (8)

$$
G(x)=\sum_{k=1}^{\infty} \frac{C_{k}}{k !} H_{k}(x) .
$$


For $z=\left(z_{1}, \ldots, z_{q}\right) \in \mathbb{R}^{q}$, we denote

$$
\sum_{i=1}^{q} z_{i} v_{i T}(t, \theta)=R_{T}(t, \theta, z)=R_{T}(t) .
$$

Then,

$$
\left\langle\zeta_{T}, z\right\rangle=\int_{0}^{T} G(\xi(t)) R_{T}(t) d t=\sum_{k=m}^{\infty} \frac{C_{k}}{k !} \int_{0}^{T} H_{m}(\xi(t)) R_{T}(t) d t,
$$

in the Hilbert space $L_{2}(\Omega, \mathbb{F}, P)$, and

$$
E\left\langle\zeta_{T}, z\right\rangle^{2}=\sum_{k=m}^{\infty} \frac{C_{k}^{2}}{k !} \int_{0}^{T} \int_{0}^{T} B^{k}(t-s) R_{T}(t) R_{T}(s) d t d s .
$$

The following condition is now assumed:

A4 Either 1) $\operatorname{Hrank}(G)=1, \alpha>1$; or 2) $\operatorname{Hrank}(G)=m \geq 2, \alpha m>1$; where $\alpha=\min _{j=0,1, \ldots, \kappa} \alpha_{j}$. In the further reasoning we use the part 2) of condition $\mathbf{A} 4$.

For $k \geq m \geq 2$, let

$$
f^{(* k)}(\lambda)=\int_{R^{k-1}} f\left(\lambda-\lambda_{2}-\cdots-\lambda_{k}\right) \prod_{i=2}^{k} f\left(\lambda_{i}\right) d \lambda_{2} \cdots d \lambda_{k},
$$

the $k$-th convolution of the spectral density given under assumption A1. Under the condition A4, $B^{k}(t) \in L_{1}(\mathbb{R}), k \geq m$. Thus, all convolutions $f^{(* k)}(\lambda), k \geq m$, are continuous and bounded functions under $\mathbf{A} 4$, and

$$
\begin{aligned}
\sigma_{T}^{2}(k, z) & =\int_{0}^{T} \int_{0}^{T} B^{k}(t-s) R_{T}(t) R_{T}(s) d t d s=\sum_{i, j=1}^{q}\left(\int_{0}^{T} \int_{0}^{T} B^{k}(t-s) v_{i T}(t, \theta) v_{j T}(s, \theta) d t d s\right) z_{i} z_{j} \\
& =2 \pi \sum_{i, j=1}^{q} \int_{\mathbb{R}} f^{(* k)}(\lambda) \mu_{T}^{i, j}(d \lambda, \theta) z_{i} z_{j} \longrightarrow 2 \pi \int_{\mathbb{R}} f^{(* k)}(\lambda) m_{z}(d \lambda, \theta)=\sigma_{k}^{2}(z),
\end{aligned}
$$

where $m_{z}(d \lambda, \theta)=\sum_{i, j=1}^{q} \mu^{i, j}(d \lambda, \theta) z_{i} z_{j}$ is a measure. Thus, as $T \longrightarrow \infty$,

$$
E\left\langle\zeta_{T}, z\right\rangle^{2} \longrightarrow 2 \pi \sum_{k=m}^{\infty} \frac{C_{k}^{2}}{k !} \int_{\mathbb{R}} f^{(* k)}(\lambda) m_{z}(d \lambda, \theta)=\sum_{k=m}^{\infty} \frac{C_{k}^{2}}{k !} \sigma_{k}^{2}(z)=\sigma^{2}(z),
$$

To prove asymptotic normality, the method of moments can be applied. That is, for any integer $p \geq 2$, it will be showed that

$$
\lim _{T \rightarrow \infty} E \eta_{T}^{p}=E \eta^{p}=\left\{\begin{array}{l}
(p-1) ! ! \sigma^{p}(z), \quad p=2 \nu, \quad \nu=1,2, \ldots, \\
0, \quad p=2 \nu+1, \quad \nu=1,2, \ldots,
\end{array}\right.
$$

where

$$
\eta_{T}=\sum_{k=m}^{\infty} \frac{C_{k}}{k !} \int_{0}^{T} H_{k}(\xi(t)) R_{T}(t) d t
$$

and $\eta \sim N\left(0, \sigma^{2}(z)\right)$. Let

$$
\eta_{T}=\eta_{T}(M)+\eta_{T}^{\prime}(M), \quad \eta_{T}(M)=\sum_{k=m}^{M} \frac{C_{k}}{k !} \int_{0}^{T} H_{k}(\xi(t)) R_{T}(t) d t .
$$


Lemma 3 Assume that the conditions $\boldsymbol{A} 1-\boldsymbol{A} 3$ and $\boldsymbol{B} 1$-B3 are satisfied and, for any $M \geq m$,

$$
\eta_{T}(M) \underset{T \rightarrow \infty}{\Longrightarrow} \eta(M) \sim N\left(0, \sigma_{M}^{2}(z)\right),
$$

where

$$
\sigma_{M}^{2}(z)=\sum_{k=m}^{M} \frac{C_{k}^{2}}{k !} \sigma_{k}^{2}(z),
$$

then,

$$
\eta_{T} \Longrightarrow \eta \sim N\left(0, \sigma^{2}(z)\right) .
$$

Proof. Note that, uniformly in $T$,

$$
E\left[\eta_{T}^{\prime}(M)\right]^{2} \longrightarrow 0, \quad M \longrightarrow \infty .
$$

Specifically, under B3, with $u=0$, for $k(0)=\left(k_{1}(0), \ldots, k_{q}(0)\right)$,

$$
\left|R_{T}(t)\right| \leq\left|\sum_{i=1}^{q} z_{i} v_{i T}(t, \theta)\right| \leq T^{-1 / 2} \sum_{i=1}^{q}\left|z_{i}\right| k_{i}(0) \leq T^{-1 / 2}\|z\| \cdot\|k(0)\| .
$$

Therefore,

$$
\begin{gathered}
E\left[\eta_{T}^{\prime}(M)\right]^{2}= \\
=E\left(\sum_{k=M+1}^{\infty} \frac{C_{k}}{k !} \int_{0}^{T} H_{k}(\xi(t)) R_{T} d t\right)^{2}=\sum_{k=M+1}^{\infty} \frac{C_{k}^{2}}{k !} \int_{0}^{T} \int_{0}^{T} B^{k}(t-s) R_{T}(t) R_{T}(s) d t d s \\
\leq\|z\|^{2}\|k(0)\|^{2} \frac{1}{T} \int_{0}^{T} \int_{0}^{T}\left|B^{m}(t-s)\right| d t d s \sum_{k=M+1}^{\infty} \frac{C_{k}^{2}}{k !} \\
\leq\|z\|^{2}\|k(0)\|^{2} \int_{-\infty}^{\infty}\left|B^{m}(t)\right| d t \sum_{k=M+1}^{\infty} \frac{C_{k}^{2}}{k !}=\pi(M) \longrightarrow 0, M \longrightarrow \infty,
\end{gathered}
$$

since $\sum_{k=m}^{\infty} \frac{C_{k}^{2}}{k !}=E G^{2}(\xi(0))<\infty$. Thus, for any $\epsilon>0$, uniformly in $T$,

$$
P\left\{\left|\eta_{T}^{\prime}(M)\right|>\epsilon\right\} \leq \frac{\pi(M)}{\epsilon^{2}} \longrightarrow 0, \quad M \longrightarrow \infty .
$$

We then obtain

$$
\begin{aligned}
P\left\{\eta_{T} \leq x\right\} & =P\left\{\eta_{T} \leq x,\left|\eta_{T}^{\prime}(M)\right| \leq \epsilon\right\}+P\left\{\eta_{T} \leq x,\left|\eta_{T}^{\prime}(M)\right|>\epsilon\right\} \\
& \leq P\left\{\eta_{T}(M)+\eta_{T}^{\prime}(M) \leq x,\left|\eta_{T}^{\prime}(M)\right| \leq \epsilon\right\}+\frac{\pi(M)}{\epsilon^{2}} \\
& \leq P\left\{\eta_{T}(M) \leq x+\epsilon\right\}+\frac{\pi(M)}{\epsilon^{2}} .
\end{aligned}
$$

Thus, we have the following

$$
\varlimsup_{T \rightarrow \infty} P\left\{\eta_{T} \leq x\right\} \leq \Phi_{M}(x+\epsilon)+\frac{\pi(M)}{\epsilon^{2}},
$$

where $\Phi_{M}$ is the d.f. of $\eta(M) \sim N\left(0, \sigma_{M}^{2}(z)\right)$.

On the other hand,

$$
\begin{aligned}
P\left\{\eta_{T}(M) \leq x-\epsilon\right\} & =P\left\{\eta_{T}(M)+\epsilon \leq x,\left|\eta_{T}^{\prime}(M)\right| \leq \epsilon\right\}+P\left\{\eta_{T}(M)+\epsilon \leq x,\left|\eta_{T}^{\prime}(M)\right|>\epsilon\right\} \\
& \leq P\left\{\eta_{T} \leq x\right\}+\frac{\pi(M)}{\epsilon^{2}}
\end{aligned}
$$


or equivalently,

$$
P\left\{\eta_{T} \leq x\right\} \geq P\left\{\eta_{T}(M) \leq x-\epsilon\right\}-\frac{\pi(M)}{\epsilon^{2}}
$$

which leads to

$$
\Phi_{M}(x-\epsilon)-\frac{\pi(M)}{\epsilon^{2}} \leq \varliminf_{T \longrightarrow \infty} P\left\{\eta_{T} \leq x\right\} .
$$

Taking the limit in (69) and (70) in $M$, we obtain that, for any $\epsilon>0$,

$$
\Phi_{\infty}(x-\epsilon) \leq \underline{\lim }_{T \rightarrow \infty} P\left\{\eta_{T} \leq x\right\} \leq \varlimsup_{T \longrightarrow \infty} P\left\{\eta_{T} \leq x\right\} \leq \Phi_{\infty}(x+\epsilon),
$$

where $\Phi_{\infty}$ is the d.f. of a normal random variable with zero mean and variance $\sigma^{2}(z)$. As $\epsilon \longrightarrow 0$, we then have the desired result.

We therefore need to prove that, for any integer $p \geq 2$, and for fixed $M \geq m$,

$$
\lim _{T \rightarrow \infty} E \eta_{T}^{p}(M)=E \eta^{p}(M)=\left\{\begin{array}{l}
(p-1) ! ! \sigma_{M}^{p}(z), \quad p=2 \nu, \quad \nu=1,2, \ldots \\
0, \quad p=2 \nu+1, \quad \nu=1,2, \ldots
\end{array}\right.
$$

where $\eta(M) \sim N\left(0, \sigma_{M}^{2}(z)\right)$. The following notation is considered:

$$
\begin{gathered}
D_{p}=\left\{J: J=\left(l_{1}, \ldots, l_{p}\right), \quad 1 \leq l_{i} \leq M, \quad i=1, \ldots, p\right\}, \\
K(J)=\prod_{i=1}^{p} \frac{C_{j_{i}}}{\left(j_{i}\right) !}, \quad \int^{(p)} \cdots=\prod_{i=1}^{p} \int_{0}^{T} \ldots
\end{gathered}
$$

Note that by diagram formula (see Lemma 1)

$$
\begin{gathered}
E \zeta_{T}^{p}(M)=E\left(\sum_{k=m}^{M} \frac{C_{k}}{k !} \int_{0}^{T} H_{k}(\xi(t)) R_{T}(t) d t\right)^{p}= \\
=\sum_{D_{p}} K(J) \int^{(p)} \prod_{j=1}^{p} R_{T}\left(t_{j}\right) \sum_{\Gamma \in L(J)} \prod_{\varpi \in R(\Gamma)} B\left(t_{d_{1}(\varpi)}-t_{d_{2}(\varpi)}\right) d t_{1} \ldots d t_{p} .
\end{gathered}
$$

Let $L^{*}(J)$ be a set of regular diagrams. We split the sum

$$
\sum_{\Gamma \in L} \cdots=\sum_{\Gamma \in L^{*}} \cdots+\sum_{\Gamma \in L \backslash L^{*}} \cdots
$$

and denote

$$
\sum_{\Gamma \in L^{*}} \ldots \equiv \sum_{p}^{*}(T), \quad \sum_{\Gamma \in L \backslash L^{*}} \ldots \equiv \sum_{p}(T) .
$$

We will study their behavior separately.

\section{Analysis of the regular diagrams:}

If $p=2 \nu+1$ is odd, then $L^{*}=\emptyset, J \in D_{p}$, and $\lim _{T \rightarrow \infty} \sum_{p}^{*}(T)=0$. If $p=2 \nu$, for an arbitrary fix regular diagram $\Gamma \in L^{*}(J), J \in D_{p}$, which has $2 m_{j}$ levels of cardinality $r_{j}$, with $m \leq r(j) \leq M$, $j=1, \ldots, l, \sum_{j=1}^{l} m_{j}=\nu$, where $1 \leq l \leq \nu$ is fixed, and all $r(j), j=1, \ldots, l$, are different, we obtain that the contribution to $\sum_{p}^{*}(T)$ is equal to

$$
\prod_{j=1}^{l}\left(\frac{C_{r(j)}}{(r(j)) !}\right)^{2 m_{j}} \sigma_{T}^{2 m_{j}}(r(j), z) \underset{T \longrightarrow \infty}{\longrightarrow} \prod_{j=1}^{l}\left(\frac{C_{r(j)}}{(r(j)) !}\right)^{2 m_{j}} \sigma^{2 m_{j}}(r(j), z) .
$$


Note that the number of regular diagrams with $2 m_{j}$ levels of cardinality $r(j), j=1, \ldots, l$, $\sum_{j=1}^{l} m_{j}=\nu$ is equal to

$$
\begin{gathered}
\frac{(2 \nu) !}{\left(2 m_{1}\right) ! \cdots\left(2 m_{l}\right) !}\left\{\prod_{j=1}^{l}\left(2 m_{j}-1\right)\left(2 m_{j}-3\right) \cdots 3 \cdot 1\right\}\left\{\prod_{j=1}^{l}(r(j) !)^{m_{j}}\right\}= \\
=\frac{(2 \nu-1) ! !(\nu) !}{m_{1} ! \ldots m_{l} !} \prod_{j=1}^{l}(r(j) !)^{m_{j}} .
\end{gathered}
$$

From (72)-(174), we obtain

$$
\begin{gathered}
\sum_{p}^{*}(T)=\sum_{J \in D_{p}} K(J) \int^{(p)} \prod_{j=1}^{p} R_{T}\left(t_{j}\right) \sum_{\Gamma \in L^{*}(J)} \prod_{\varpi \in R(\Gamma)} B\left(t_{d_{1}(\varpi)}-t_{d_{2}(\varpi)}\right) d t_{1} \ldots d t_{p} \\
=(2 \nu-1) ! ! \sum_{1<l<\nu} \sum_{\substack { m_{1}+\ldots+m_{l}=\nu \\
\begin{subarray}{c}{m \leq r(j) \leq M \\
1 \leq j \leq l{ m _ { 1 } + \ldots + m _ { l } = \nu \\
\begin{subarray} { c } { m \leq r ( j ) \leq M \\
1 \leq j \leq l } }\end{subarray}} \frac{\nu !}{m_{1} ! \ldots m_{l} !} \prod_{j=1}^{l}\left[\frac{C_{r(j)}^{2}}{r(j) !} \sigma_{T}^{2}(r(j), z)\right]^{m_{j}}= \\
=(p-1) ! !\left[\sum_{r(j)=m}^{M} \frac{C_{r(j)}^{2}}{r(j) !} \sigma_{T}^{2}(r(j), z)\right]_{T \longrightarrow \infty}^{\frac{p}{2}}(p-1) ! !\left[\sum_{r(j)=m}^{M} \frac{C_{r(j)}^{2}}{r(j) !} \sigma^{2}(r(j), z)\right]^{\frac{p}{2}}= \\
=(p-1) ! ! \sigma_{M}^{p}(z) .
\end{gathered}
$$

Analysis of the nonregular diagrams:

We now wish to prove that

$$
\sum_{p}(T)=\sum_{J \in D_{p}} K(J) \sum_{\Gamma \in L \backslash L^{*}} I_{\Gamma}(J, T) \longrightarrow 0
$$

where

$$
I_{\Gamma}(J, T)=\int^{(p)} \prod_{j=1}^{p} R_{T}\left(t_{j}\right) \prod_{\varpi \in R(\Gamma)} B\left(t_{d_{1}(\varpi)}-t_{d_{2}(\varpi)}\right) d t_{1} \ldots d t_{p} .
$$

Now, we assume that the diagram $\Gamma^{\prime} \in L\left(l_{1}^{\prime}, \ldots, l_{p}^{\prime}\right)$ satisfies $l_{1}^{\prime} \leq \cdots \leq l_{p}^{\prime}$. We then have

$$
\left|I_{\Gamma}(J, T)\right| \leq\|z\|^{p}\|k(0)\|^{p} T^{-p / 2} \int^{(p)} \prod_{\substack{\varpi \in R(\Gamma) \\ d_{1}(\varpi)=j}} B\left(t_{j}-t_{d_{2}(\varpi)}\right) d t_{1} \ldots d t_{p} .
$$

Given a permutation $\pi$ of the set $(1, \ldots, p)$ and the diagram $\Gamma \in L\left(l_{1}, \ldots, l_{p}\right)$, we define the diagram $\pi \Gamma$ in the following way: the $\pi(j)$ level of $\pi \Gamma$ has cardinality $l_{j}, j=1, \ldots, p$, and $\varpi=\left(\left(j_{1}, k_{1}\right),\left(j_{2}, k_{2}\right)\right) \in$ $R(\Gamma)$ if and only if $\pi(\varpi)=\left(\left(\pi\left(j_{1}\right), k_{1}\right),\left(\pi\left(j_{2}\right), k_{2}\right)\right) \in \pi R(\Gamma)$. Given a diagram $\Gamma \in L\left(l_{1}, \ldots, l_{p}\right)$, we define the integer-valued function $q_{\Gamma}(j)$ on the set $\{1, \ldots, p\}$ in the following way: $q_{\Gamma}(j)$ is the cardinality of the edges $\varpi \in R(\Gamma)$ such that $d_{1}(\varpi)=j$.

Observe that for $\Gamma \in L\left(l_{1}, \ldots, l_{p}\right)$, and $J=\left(l_{1}, \ldots, l_{p}\right)$

$$
I_{\Gamma}(J, T)=I_{\pi \Gamma}(\pi J, T), \quad \pi J=\left(l_{\pi^{-1}(1)}, \ldots, l_{\pi^{-1}(p)}\right) .
$$

For all the diagrams $\Gamma$, there exists a permutation $\pi$ such that $\Gamma^{\prime}=\pi \Gamma$ has the following property: $\Gamma^{\prime} \in L\left(l_{1}^{\prime}, \ldots, l_{p}^{\prime}\right)$ and

$$
l_{1}^{\prime} \leq \cdots \leq l_{p}^{\prime}
$$


Then, for $q_{\Gamma}(j) \geq 1$,

$$
\begin{gathered}
\int_{0}^{T} \prod_{\substack{\varpi \in R(\Gamma) \\
d_{1}(\varpi)=j}} B\left(t_{j}-t_{d_{2}(\varpi)}\right) d t_{j} \leq \frac{1}{q_{\Gamma}(j)} \sum_{\substack{\varpi \in R(\Gamma) \\
d_{1}(\varpi)=j}} \int_{0}^{T}\left|B\left(t_{j}-t_{d_{2}(\varpi)}\right)\right|^{q_{\Gamma}(j)} d t_{j} \leq \\
\leq 2 \int_{0}^{T}\left|B\left(t_{j}\right)\right|^{q_{\Gamma}(j)} d t_{j} .
\end{gathered}
$$

If $q_{\Gamma}(i)=0$, the integrals regarded to these variables, after (80), give a contribution in the form of multiplier $T$ in the estimate (777).

Definition 1 The level $j$ of a nonregular diagram $\Gamma \in L \backslash L^{*}$ is said to be a donor, if $q_{\Gamma}(j) \geq 1$, and a strong donor, if $q_{\Gamma}(j)=l_{j}$. The level $j$ of a nonregular diagram $\Gamma \in L \backslash L^{*}$ is said to be a recipient, if it is not donor, that is, $q_{\Gamma}(j)=0$.

Let $\rho_{s d}$ be a number of strong donor levels, and $\rho_{r}$ is a number of recipient levels. Note that $\rho_{s d}, \rho_{r} \geq 1$, since the level 1 is a strong donor one, while the level $p$ is a recipient one.

Formulae (77) and (80) now imply

$$
\left|I_{\Gamma}(J, T)\right| \leq 2^{p-\rho_{r}}\|z\|^{p}\|k(0)\|^{p} T^{-p / 2} \prod_{j=1}^{p} \int_{0}^{T}|B(t)|^{q_{\Gamma}(j)} d t .
$$

Since $l_{j} \geq m \geq 2$, under $\mathbf{B 6}$, for a strong donor level $j$

$$
\int_{0}^{T}|B(t)|^{l_{j}} d t \leq \int_{0}^{\infty}[B(t)]^{2} d t<\infty .
$$

Thus, for the recipient levels $\left(q_{\Gamma}(j)=0\right)$, and the strong donor levels $\left(q_{\Gamma}(j)=l_{j}\right)$, we obtain

$$
\int_{0}^{T}|B(t)|^{q_{\Gamma}(j)} d t \leq c_{0} T^{1-z(j)}
$$

where $z(j)=\frac{q_{\Gamma}(j)}{l_{j}}, c_{0}=\max \left(1, \int_{0}^{\infty} B(t)^{2} d t\right)$. Let now $0<q_{\Gamma}(j)<l_{j}$, that is, a level $j$ is a donor one, but not strong donor. Since $\int_{0}^{\infty}|B(t)|^{l_{j}} d t<\infty$, for any $\epsilon>0$, there exists $T_{\epsilon}$, such that $\int_{T_{\epsilon}}^{\infty}|B(t)|^{l_{j}} d t<\epsilon$. Hence, it follows from the Hölder inequality $\left(\frac{1}{p}=z(j)=\frac{q_{\Gamma}(j)}{l_{j}}, \frac{1}{q}=1-z(j)\right)$, that for sufficiently large $T$

$$
\begin{gathered}
\int_{0}^{T}|B(t)|^{q_{\Gamma}(j)} d t=\int_{0}^{T_{\epsilon}}|B(t)|^{q_{\Gamma}(j)} d t+\int_{T_{\epsilon}}^{T}|B(t)|^{q_{\Gamma}(j)} d t \leq \\
\leq C(\epsilon)+\left(\int_{T_{\epsilon}}^{T}\left[|B(t)|^{q_{\Gamma}(j)}\right]^{\frac{l_{j}}{q_{\Gamma}(j)}} d t\right)^{z(j)} T^{1-z(j)} \leq \\
\leq C(\epsilon)+\epsilon^{z(j)} T^{1-z(j)}=o\left(T^{1-z(j)}\right) .
\end{gathered}
$$

Denoting by $\mu=\frac{p}{2}-\sum_{j=1}^{p} z(j)$, formulae (83) and (84) together with (81) lead to

$$
\left|I_{\Gamma}(J, T)\right|=O\left(T^{\mu}\right), \quad T \longrightarrow \infty,
$$

if all the levels of $\Gamma$ are strong donor and recipient, and

$$
\left|I_{\Gamma}(J, T)\right|=o\left(T^{\mu}\right), \quad T \longrightarrow \infty,
$$


if $0<q_{\Gamma}(j)<l_{j}$, for some $j$.

Let us show that $\mu \leq 0$. Choose an edge $\varpi \in R(\Gamma)$, and define the numbers $p_{1}(\varpi)$ and $p_{2}(\varpi)$ as the cardinalities of levels $d_{1}(\varpi)$ and $d_{2}(\varpi)$ respectively. Observe that $p_{1}(\varpi) \leq p_{2}(\varpi)$, for any $\varpi \in R(\Gamma)$. Taking into account the definition of the $z(j)$, we obtain

$$
2 \sum_{j=1}^{p} z(j)=2 \sum_{j=1}^{p} \frac{q_{\Gamma}(j)}{l_{j}}=2 \sum_{\varpi \in R(\Gamma)} \frac{1}{p_{1}(\varpi)} \geq \sum_{\varpi \in R(\Gamma)}\left[\frac{1}{p_{1}(\varpi)}+\frac{1}{p_{2}(\varpi)}\right]=p,
$$

because the term $1 / l_{i}$ appears exactly $l_{i}$ times among the summands $1 / p_{1}(\varpi)$ and $1 / p_{2}(\varpi)$. The following inequality then holds

$$
\sum_{1 \leq i \leq p} z(i) \geq \frac{p}{2}, \quad \text { or } \quad \mu \leq 0,
$$

where there is a strict inequality if $\Gamma$ contains an edge connecting levels of different cardinality.

Thus, if in $\Gamma=\Gamma\left(l_{1}, \ldots, l_{p}\right) \in L \backslash L^{*}, l_{1} \leq \cdots \leq l_{p}$, there is an edge between levels of different cardinalities, and all the levels are strongly donor or recipient ones, then, from (85),

$$
\left|I_{\Gamma}(J, T)\right| \longrightarrow 0, \quad T \longrightarrow \infty,
$$

while if there is level $j$ such that $0<q_{\Gamma}(j)<l_{j}$, then (89) follows from (86) and (88).

We assume now that all edges of a non-regular diagram $\Gamma=\Gamma\left(l_{1}, \ldots, l_{p}\right) \in L \backslash L^{*}, l_{1} \leq \cdots \leq l_{p}$, connect the levels of the same cardinality. To complete the proof one can use the following observations: Let $i$ be the first upper recipient level. If it got an edge from the one (strongly donor) level upper it, then the integral on variable $t_{j}$ in the right hand size of (81) can be estimated by a constant, while the integral on the variable corresponding to the above strongly donor level can be estimated by $T$. Then, one can remove these levels from the consideration. Thus, we can consider the moment of order $(p-2)$ (instead of order $p$ ). Since the diagram $r$ is nonregular, one can continue the above procedure until the case where the recipient level got edges from more than one donor level upper it.

Let $i$ be the first upper recipient level that has edges from at least two donor's levels $j$ and $k$ upper it, $j<k<i$, and $k$ is the nearest to $i$ donor level. Level $k$ does not give all edges to $i$.

Let us change $k$ and $i$, and denote this permutation by $\tilde{\pi}$. Then, $\tilde{\pi}(k)=i, \tilde{\pi}(i)=k, \tilde{\pi}(i)<\tilde{\pi}(k)$, and from the level $\tilde{\pi}(i)$ to $\widetilde{\pi}(k)$ will enter less than $l_{i}=l_{k}=l$ edges. Moreover, $q_{\tilde{\pi} \Gamma}(\tilde{\pi}(i))=q_{\tilde{\pi} \Gamma}(k)<l$, since the only down edges from $\tilde{\pi}(i)$ are those connecting $\tilde{\pi}(i)$ with $\tilde{\pi}(k)$.

Let nonregular diagram $\Gamma=\Gamma\left(l_{1}, \ldots, l_{p}\right), l_{1} \leq \cdots \leq l_{p}$, does not contain any donor level $j$ such that $q_{\Gamma}(j)<l_{j}$. Then, the following dichotomy holds: either $\Gamma$ connects the levels of different cardinalities, or there exists a permutation of a strongly donor (say, $k$-th), and recipient levels, such that $0<q_{\tilde{\pi} \Gamma}(k)<l$, where $l$ is the joint cardinality of both levels. Thus, we have proven (76) , i.e., the following statement holds:

Theorem 4 Under conditions $\boldsymbol{A} \mathbf{1 - A 4}, \mathbf{B 1 - B 3}$ and $\mathbf{B 6}$, the random vector (56) converges in distribution, as $T \longrightarrow \infty$, to the Gaussian vector $N(0, \Sigma)$, where

$$
\Sigma=2 \pi \sum_{k=m}^{\infty} \frac{C_{k}^{2}}{k !} \int_{\mathbb{R}} f^{(* k)}(\lambda) \boldsymbol{\mu}(d \lambda, \theta)
$$

with $\boldsymbol{\mu}(d \lambda, \theta)$ being the spectral measure of the regression function, and $f^{(* k)}(\lambda)$ being the $k$ th selfconvolution of s.d. under assumption $\boldsymbol{A} \mathbf{1}$.

Now we are able to prove the asymptotic normality of the LSE .

Theorem 5 Assume that conditions $\boldsymbol{A} 1-\boldsymbol{A} 4, B 1-B 6$ and $\boldsymbol{C}$ hold. Then, the random vector $\hat{u}_{T}=$ $d_{T}(\theta)\left(\hat{\theta}_{T}-\theta\right)$ converges, in distribution, to the Gaussian vector $N\left(0, \Sigma_{0}\right)$, as $T \longrightarrow \infty$, where

$$
\Sigma_{0}=2 \pi \sum_{k=m}^{\infty} \frac{C_{k}^{2}}{k !}\left(\int_{\mathbb{R}} \boldsymbol{\mu}(d \lambda, \theta)\right)^{-1}\left(\int_{\mathbb{R}} f^{(* k)}(\lambda) \boldsymbol{\mu}(d \lambda, \theta)\right)\left(\int_{\mathbb{R}} \boldsymbol{\mu}(d \lambda, \theta)\right)^{-1} .
$$


Proof. In the notation of Sections 4 and 5, we obtain

$$
L_{T}^{i}(u)=\int_{0}^{T}\left(G(\xi(t))-\sum_{l=1}^{q} v_{l T}(t, \theta) u_{l}\right) v_{i T}(t, \theta) d t=0, \quad i=1, \ldots, q,
$$

or equivalently,

$$
\begin{aligned}
\int_{0}^{T} G(\xi(t)) v_{i T}(t, \theta) d t & =\int_{0}^{T} \sum_{l=1}^{q} v_{l T}(t, \theta) u_{l} v_{i T}(t, \theta) d t \\
\sum_{l=1}^{q} \int_{0}^{T} v_{l T}(t, \theta) v_{i T}(t, \theta) d t u_{l} & =\sum_{l=1}^{q} J_{i l, T}(\theta) u_{l}=\int_{0}^{T} G(\xi(t)) v_{i T}(t, \theta) d t .
\end{aligned}
$$

Thus, we have a system of equations regarded to $u: J_{T}(\theta) u=d_{T}^{-1}(\theta) \int_{0}^{T} G(\xi(t)) \nabla g(t, \theta) d t$, or $\widetilde{u}_{T}=\left(\widetilde{u}_{1}, \ldots, \widetilde{u}_{q}\right)^{\prime}=\Lambda_{T}(\theta) \int_{0}^{T} G(\xi(t)) d_{T}^{-1}(\theta) \nabla g(t, \theta) d t=\Lambda_{T}(\theta) \zeta_{T}$, where $\Lambda_{T}(\theta)=J_{T}^{-1}(\theta), d_{T}(\theta)=$ $\operatorname{diag}\left(d_{i T}(\theta)\right)_{i=1}^{q}, \nabla g(t, \theta)=\left(g_{1}(t, \theta), \ldots, g_{q}(t, \theta)\right)^{\prime}$.

From Theorem 4 the vector

$$
\tilde{u}_{T}=\Lambda_{T}(\theta)\left(\int_{0}^{T} \varepsilon(t) v_{i T}(t, \theta) d t\right)_{i=1}^{q}
$$

is asymptotically normal. To compute the limiting covariance matrix, we note that the covariance matrix of the vector $\tilde{u}_{T}$ has the form $\Sigma_{0 T}=\Lambda_{T}(\theta) \sigma_{T}^{2}(\theta) \Lambda_{T}(\theta)$, where $\sigma_{T}^{2}(\theta)$ is covariance matrix of the vector $\zeta_{T}$. As $T \longrightarrow \infty$,

$$
\begin{aligned}
\Sigma_{0 T} & =2 \pi \sum_{k=m}^{\infty} \frac{C_{k}^{2}}{k !}\left(\int_{\mathbb{R}} \boldsymbol{\mu}_{T}(d \lambda, \theta)\right)^{-1}\left(\int_{\mathbb{R}} f^{(* k)}(\lambda) \boldsymbol{\mu}_{T}(d \lambda, \theta)\right)\left(\int_{\mathbb{R}} \boldsymbol{\mu}_{T}(d \lambda, \theta)\right)^{-1} \\
& \longrightarrow 2 \pi \sum_{k=m}^{\infty} \frac{C_{k}^{2}}{k !}\left(\int_{\mathbb{R}} \boldsymbol{\mu}(d \lambda, \theta)\right)^{-1}\left(\int_{\mathbb{R}} f^{(* k)}(\lambda) \boldsymbol{\mu}(d \lambda, \theta)\right)\left(\int_{\mathbb{R}} \boldsymbol{\mu}(d \lambda, \theta)\right)^{-1}=\Sigma_{0} .
\end{aligned}
$$

We need to prove that the d.f. $F_{T}(y, \theta)$ of the vector $\hat{u}_{T}=d_{T}(\theta)\left(\hat{\theta}_{T}-\theta\right)$ converges to the Gaussian d.f. $\Phi_{0, \Sigma_{0}}(y)$ as $T \longrightarrow \infty$.

Then, we will show that, for any $r>0$,

$$
\Delta_{T}(r)=P\left\{\left\|\hat{u}_{T}-\tilde{u}_{T}\right\|>r\right\} \longrightarrow 0, T \longrightarrow \infty .
$$

Denote the event $A_{T}=\left\{\tilde{u}_{T} \in V^{c}(R-r)\right\}$, where $R$ is such that, for $T>T_{0}, P\left(\bar{A}_{T}\right)<\frac{\epsilon}{2}$, for a fixed $\epsilon>0$. This follows from the asymptotic normality of $\tilde{u}_{T}$. Introduce one more event $B_{T}=\left\{\sup _{u \in V^{c}(R)}\left\|\Lambda_{T}(\theta)\left(\psi_{T}(u)-L_{T}(u)\right)\right\|\right\} \leq r$. From Theorem 3 , we obtain that, for $T>T_{0}$,

$$
\begin{aligned}
P\left(B_{T}\right) & =P\left\{\sup _{u \in V^{c}(R)}\left\|\Lambda_{T}(\theta)\left(\psi_{T}(u)-L_{T}(u)\right)\right\|>r\right\} \leq P\left\{\lambda_{\max }\left(\Lambda_{T}(\theta)\right) \sup _{u \in V^{c}(R)}\left\|\psi_{T}(u)-L_{T}(u)\right\|>r\right\} \\
& =P\left\{\frac{1}{\lambda_{\min }\left(J_{T}(\theta)\right)} \sup _{u \in V^{c}(R)}\left\|\psi_{T}(u)-L_{T}(u)\right\|>r\right\} \leq P\left\{\frac{1}{\lambda_{*}} \sup _{u \in V^{c}(R)}\left\|\psi_{T}(u)-L_{T}(u)\right\|>r\right\} \\
& =P\left\{\sup _{u \in V^{c}(R)}\left\|\psi_{T}(u)-L_{T}(u)\right\|>\lambda_{*} r\right\} \leq \frac{\epsilon}{3} .
\end{aligned}
$$

Introduce also the event $C_{T}=\left\{\mathrm{LSE} \hat{u}_{T}\right.$ is unique solution of the system (48) $\}$. From Theorem 3, consider $T>T_{0}$ such that $P\left\{\bar{C}_{T}\right\} \leq \frac{\epsilon}{3}$. Thus, for $T>T_{0}$,

$$
P\left(A_{T} \cap B_{T} \cap C_{T}\right) \geq 1-\epsilon .
$$


Then,

$$
\begin{aligned}
\Lambda_{T}(\theta) L_{T}(u) & =\Lambda_{T}(\theta)\left(\int_{0}^{T} \varepsilon(t) v_{i T}(t, \theta) d t\right)_{i=1}^{q}-\Lambda_{T}(\theta)\left(\sum_{l=1}^{q} u_{l} \int_{0}^{T} v_{l T}(t, \theta) v_{i T}(t, \theta) d t\right)_{i=1}^{q} \\
& =\tilde{u}_{T}-\Lambda_{T}(\theta)\left(\sum_{l=1}^{q} u_{l} J_{i l, T}(\theta)\right)_{i=1}^{q}=\tilde{u}_{T}-u .
\end{aligned}
$$

For $u \in V^{c}(R)$, under event $A_{T} \cap B_{T} \cap C_{T}$, we have

$$
\begin{aligned}
\left\|u+\Lambda_{T}(\theta) \psi_{T}(u)\right\| & =\left\|u+\Lambda_{T}(\theta)\left(\psi_{T}(u)-L_{T}(\theta)\right)+\Lambda_{T}(\theta) L_{T}(u)\right\| \\
& =\left\|u+\tilde{u}_{T}-u+\Lambda_{T}(\theta)\left(\psi_{T}(u)-L_{T}(\theta)\right)\right\| \leq\left\|\tilde{u}_{T}\right\|+\| \Lambda_{T}(\theta)\left(\psi_{T}(u)-L_{T}(\theta) \|\right. \\
& \leq R-r+r=R,
\end{aligned}
$$

that is,

$$
F_{T}(u)=u+\Lambda_{T}(\theta)\left(\psi_{T}(u)\right): V^{c}(R) \rightarrow V^{c}(R)
$$

is a continuous map.

To prove (94) we will apply Fix Point Brouwer Theorem (Milnor (1965), p. 14). Specifically, if $F: V^{c}(R) \rightarrow V^{c}(R)$ is a continuous map, then, there exists $x_{0} \in V^{c}(R)$ such that $F\left(x_{0}\right)=x_{0}$. From Brouwer Theorem, there exists $u_{T}^{0} \in V^{C}(R)$ such that $F_{T}\left(u_{T}^{0}\right)=u_{T}^{0}$, and hence, $\psi_{T}\left(u_{T}^{0}\right)=0$, since $\Lambda_{T}(\theta)$ is non degenerated.

Under $C_{T}$, the normed LSE $\hat{u}_{T}$ is the unique solution to the equation

$$
\psi_{T}(u)=0, \quad u \in V^{c}(R) .
$$

Thus, $A_{T} \cap B_{T} \cap C_{T} \subset\left\{\hat{u}_{T} \in V^{c}(R)\right\}$ and $P\left\{\hat{u}_{T} \in V^{c}(R)\right\} \geq 1-\epsilon$. From (95), we get

$$
\begin{aligned}
1-\epsilon & \leq P\left\{\left\{\hat{u}_{T} \in V^{c}(R)\right\} \cap B_{T}\right\}=P\left\{\left\{\hat{u}_{T} \in V^{c}(R)\right\} \cap\left\{\sup _{u \in V^{c}(R)}\left\|\Lambda_{T}(\theta)\left(\psi_{T}(u)-L_{T}(u)\right)\right\| \leq r\right\}\right\} \\
& \leq P\left\{\left\|\Lambda_{T}(\theta)\left(\psi_{T}(\hat{u})-L_{T}(\hat{u})\right)\right\| \leq r\right\}=P\left\{\left\|\Lambda_{T}(\theta) L_{T}(\widehat{u})\right\| \leq r\right\}=P\left\{\left\|\tilde{u}_{T}-\hat{u}_{T}\right\| \leq r\right\} .
\end{aligned}
$$

Therefore, (94) follows from (96).

Let us consider the notation

$$
\Pi(-\infty ; y \pm \vec{\epsilon})=\left(-\infty ; y_{1} \pm \epsilon\right) \times \cdots \times\left(-\infty ; y_{q} \pm \epsilon\right), \quad \epsilon \geq 0 .
$$

For the d.f. $F_{T}(y, \theta)=P\left\{\tilde{u}_{T} \in \Pi(-\infty ; y)\right\}$, we obtain from (94),

$$
F_{T}(y, \theta) \geq P\left\{\tilde{u}_{T} \in \Pi(-\infty ; y-\vec{\epsilon})\right\}-\Delta_{T}(\epsilon), F_{T}(y, \theta) \leq P\left\{\tilde{u}_{T} \in \Pi(-\infty ; y-\vec{\epsilon})\right\}+\Delta_{T}(\epsilon),
$$

for any $y \in \mathbb{R}^{q}$, and $\epsilon>0$. We know that

$$
\left|P\left\{\tilde{u}_{T} \in \Pi(-\infty ; y \pm \vec{\varepsilon})\right\}-\Phi_{0, \gamma(\theta)}(y \pm \vec{\varepsilon})\right| \longrightarrow 0, \quad T \longrightarrow \infty .
$$

Let $\phi(y, \theta)$ be the probability density function of a Gaussian random variable with d.f. $\Phi_{0, \gamma(\theta)}(y)$. Since $\lambda_{\min }(\gamma(\theta))=\underline{\lambda}>0, \lambda_{\max }(\gamma(\theta))=\bar{\lambda}<\infty$, then

$$
\phi(y, \theta) \leq(2 \pi \underline{\lambda})^{-q / 2} \exp \left\{\frac{-\|y\|^{2}}{2 \bar{\lambda}}\right\}=\nu(\|y\|) .
$$

For $\mathcal{A} \in \mathcal{B}^{q}$, with $\mathcal{B}^{q}$ being the $\sigma$-algebra of Borel sets of $\mathbb{R}^{q}$, and for $\epsilon>0$, let

$$
A_{\epsilon}=\left\{x \in \mathbb{R}^{q}: \inf _{y \in \mathcal{A}}\|x-y\|<\epsilon\right\}, \quad A_{-\epsilon}=\mathbb{R}^{q} \backslash\left(\mathbb{R}^{q} \backslash A\right)_{\epsilon} .
$$

If $A=\Pi(-\infty ; y)$, then, $A_{-\epsilon}=\Pi(-\infty ; y-\vec{\epsilon}), \Pi(-\infty ; y+\vec{\epsilon})_{-\epsilon}=\mathbb{R}^{q} \backslash \Pi(-\infty ; y)=A^{c}$. We will apply Theorem $\S 3$ of Bhattacharya and Ranga Rao (1976). 
Lemma 4 Let $\nu$ be a non-negative differential function on $[0, \infty)$, such that

(1) $b=\int_{0}^{\infty}\left|\nu^{\prime}(\lambda)\right| \lambda^{q-1} d \lambda<\infty$;

(2) $\lim _{\lambda \rightarrow \infty} \nu(\lambda)=0$.

Then for any convex $C \in \mathcal{B}^{q}$ and given $\epsilon, \delta>0$, we have

$$
\int_{C_{\epsilon} \backslash C_{-\delta}} \nu(\|\lambda\|) d \lambda \leq b\left(\frac{2 \pi^{q / 2}}{\Gamma\left(\frac{q}{2}\right)}\right)(\epsilon+\delta) .
$$

From Lemma 4 , for any $\psi \neq 0$, we have

$$
\left|\Phi_{0, \gamma(\theta)}(y)-\Phi_{0, \gamma(\theta)}(y+\vec{\psi})\right|=\int_{\Pi} \phi(y, \theta) d y \leq b\left(\frac{2 \pi^{q / 2}}{\Gamma\left(\frac{q}{2}\right)}\right)|\psi|,
$$

where $\Pi= \begin{cases}\Pi(-\infty, y+\vec{\psi}) \backslash A^{c}, & \psi>0, \\ A \backslash A_{\psi}, & \psi<0 .\end{cases}$

For any $y \in \mathbb{R}^{q}$, and $\epsilon>0$,

$$
\begin{aligned}
F_{T}(y, \theta)-\Phi_{0, \gamma(\theta)}(y) & \leq \Delta_{T}(\epsilon)+P\left\{\tilde{u}_{T} \in \Pi(-\infty ; y+\vec{\epsilon})\right\}-\Phi_{0, \gamma(\theta)}(y) \\
& \leq \Delta_{T}(\epsilon)+\left|P\left\{\tilde{u}_{T} \in \Pi(-\infty ; y+\vec{\epsilon})\right\}-\Phi_{0, \gamma(\theta)}(y)\right| \\
& \leq \Delta_{T}(\epsilon)+\left|P\left\{\tilde{u}_{T} \in \Pi(-\infty ; y+\vec{\epsilon})\right\}-\Phi_{0, \gamma(\theta)}(y+\vec{\epsilon})\right|+\left|\Phi_{0, \gamma(\theta)}(y+\vec{\epsilon})-\Phi_{0, \gamma(\theta)}(y)\right| ; \\
\Phi_{0, \gamma(\theta)}(y)-F_{T}(y, \theta) & \leq \Delta_{T}(\epsilon)-P\left\{\tilde{u}_{T} \in \Pi(-\infty ; y-\vec{\epsilon})\right\}+\Phi_{0, \gamma(\theta)}(y) \\
& \leq \Delta_{T}(\epsilon)+\left|\Phi_{0, \gamma(\theta)}(y)-P\left\{\tilde{u}_{T} \in \Pi(-\infty ; y-\vec{\epsilon})\right\}\right| \\
& \leq \Delta_{T}(\epsilon)+\left|\Phi_{0, \gamma(\theta)}(y-\vec{\epsilon})-P\left\{\tilde{u}_{T} \in \Pi(-\infty ; y-\vec{\epsilon})\right\}\right|+\left|\Phi_{0, \gamma(\theta)}(y)-\Phi_{0, \gamma(\theta)}(y-\vec{\epsilon})\right| .
\end{aligned}
$$

Therefore, we have

$$
\left|F_{T}(y, \theta)-\Phi_{0, \gamma(\theta)}(y)\right| \longrightarrow 0, T \longrightarrow \infty .
$$

Thus, Theorem 5 is proven.

\section{Asymptotic normality of the LSE of the parameters of trigono- metric regression}

The asymptotic Gaussian distribution of the LSE in the Walker sense of the regression function (3) is established in the following result.

Theorem 6 Under conditions A1-A4, the LSE in the Walker sense of the function (3) of unknown parameter is asymptotically normal, that is, the vector

$$
\left(T^{1 / 2}\left(\hat{A}_{k T}-A\right), T^{1 / 2}\left(\hat{B}_{k T}-B\right), T^{3 / 2}\left(\hat{\varphi}_{k T}-\varphi\right), k=1, \ldots, N\right)
$$

converges weakly to the multidimensional normal vector $N_{3 N}(0, \Gamma)$, where the matrix $\Gamma>0$ is of the form $\Gamma=\operatorname{diag}\left(\Gamma_{k}\right)_{k=1}^{N}$, with

$$
\Gamma_{k}=\frac{4 \pi}{A_{k}^{2}+B_{k}^{2}} \sum_{j=m}^{\infty} \frac{C_{j}^{2}}{j !} f^{(* j)}\left(\varphi_{k}\right)\left(\begin{array}{ccc}
A_{k}^{2}+B_{k}^{2} & -3 A_{k} B_{k} & -6 B_{k} \\
-3 A_{k} B_{k} & A_{k}^{2}+B_{k}^{2} & 6 A_{k} \\
-6 B_{k} & 6 A_{k} & 12
\end{array}\right) .
$$

Here, $f^{(* j)}(\lambda), \lambda \in \mathbb{R}$, is the $j$-th convolution of the spectral density given under assumption $\boldsymbol{A} \mathbf{1}$. 
The spectral measures of the trigonometric regression were investigated, for example, by Whittle (1952), Walker (1973) and Ivanov (1980) (see also the monograph by Quinn and Hannan, 2001). Theorem 6 follows from the results of Section 5 by direct computations. Indeed, for the nonlinear function (3), the spectral measure $\mu(d \lambda, \theta)=\operatorname{diag}\left(\tilde{\Gamma}_{k}\right)_{k=1}^{N}$, where

$$
\tilde{\Gamma}_{k}=\left(\begin{array}{ccc}
\delta_{k} & i \rho_{k} & \bar{\beta}_{k} \\
i \rho_{k} & \delta_{k} & \bar{\gamma}_{k} \\
\beta_{k} & \gamma_{k} & \delta_{k}
\end{array}\right), \quad \beta_{k}=\frac{\sqrt{3}\left(B_{k} \delta_{k}+i A_{k} \rho_{k}\right)}{2 \sqrt{A_{k}^{2}+B_{k}^{2}}}, \quad \gamma_{k}=\frac{\sqrt{3}\left(-A_{k} \delta_{k}-i B_{k} \rho_{k}\right)}{2 \sqrt{A_{k}^{2}+B_{k}^{2}}}
$$

with the measure $\delta_{k}=\delta_{k}(d \lambda)$, and the signed measure $\rho_{k}=\rho_{k}(d \lambda)$ being located at the points $\pm \varphi_{k}$, $k=1, \ldots, N$. Here, $\delta_{k}\left(\left\{ \pm \varphi_{k}\right\}\right)=\frac{1}{2} ; \rho_{k}\left(\left\{ \pm \varphi_{k}\right\}\right)= \pm \frac{1}{2}, k=1, \ldots, N$. Thus, $\Sigma_{0}=\operatorname{diag}\left(\Sigma_{0 k}\right)_{k=1}^{N}$, where

$$
\Sigma_{0 k}(\theta)=2 \pi \sum_{j=m}^{\infty} \frac{C_{j}^{2}}{j !} f^{(* j)}\left(\varphi_{k}\right)\left(\begin{array}{ccc}
1 & 0 & \frac{B_{k}}{\sqrt{\frac{3}{4}\left(A_{k}^{2}+B_{k}^{2}\right)}} \\
0 & 1 & \frac{-A_{k}}{\sqrt{\frac{3}{4}\left(A_{k}^{2}+B_{k}^{2}\right)}} \\
\frac{B_{k}}{\sqrt{\frac{3}{4}\left(A_{k}^{2}+B_{k}^{2}\right)}} & \frac{-A_{k}}{\sqrt{\frac{3}{4}\left(A_{k}^{2}+B_{k}^{2}\right)}} & 1
\end{array}\right)^{-1}
$$

and direct computations complete the proof.

\section{Remarks on some future development}

To make Theorem 4 operational, some estimation results to approximate the limiting variance in (90) should be needed. In general this problem deserves a separate publication, but in short one can use the following arguments.

Consider the block-diagonal covariance matrix $\Gamma$ in equation (99). Let us take for its blocks $\Gamma_{k}$ their statistical estimators $\widehat{\Gamma}_{k}$ substituting into $\Gamma_{k}$ the $\operatorname{LSE}\left(A_{k T}, B_{k T}, \varphi_{k T}\right)$ instead of unknown parameters $\left(A_{k}, B_{k}, \varphi_{k}\right)$.

Theorem 7 Under conditions $\boldsymbol{A} 1$ - $A$ 4, $\widehat{\Gamma}_{k} \stackrel{P}{\longrightarrow} \Gamma_{k}, T \longrightarrow \infty, k=1, \ldots, N$.

Proof. Using the notation $B_{m}=\int_{-\infty}^{\infty}|B(t)|^{m} d t<\infty$, for $j \geq m$, obviously,

$$
\begin{aligned}
\left|f^{(* j)}\left(\widehat{\varphi}_{k T}\right)\right| & =\frac{1}{2 \pi}\left|\int_{-\infty}^{\infty} B^{j}(t) \cos \left(\widehat{\varphi}_{k T} t\right) d t\right| \leq \frac{1}{2 \pi} B_{m} \\
\left|f^{(* j)}\left(\widehat{\varphi}_{k T}\right)-f^{(* j)}\left(\varphi_{k T}\right)\right| & =\frac{1}{2 \pi}\left|\int_{-\infty}^{\infty} B^{j}(t)\left[\cos \left(\widehat{\varphi}_{k T} t\right)-\cos \left(\varphi_{k T} t\right)\right] d t\right| \\
& \leq \frac{1}{2 \pi} \int_{-T}^{T}|B(t)|^{j}\left|\cos \left(\widehat{\varphi}_{k T} t\right)-\cos \left(\varphi_{k T} t\right)\right| d t+\frac{2}{T} \int_{T}^{\infty}|B(t)|^{j} d t \\
& \leq \frac{B_{m}}{2 \pi} T\left|\widehat{\varphi}_{k T}-\varphi_{k}\right|+\frac{2}{T} \int_{T}^{\infty}|B(t)|^{m} d t .
\end{aligned}
$$

Inequality (101) shows that for $j \geq m, k=1, \ldots, N$,

$$
f^{(* j)}\left(\widehat{\varphi}_{k T}\right) \stackrel{P}{\longrightarrow} f^{(* j)}\left(\varphi_{k T}\right), \quad T \longrightarrow \infty
$$

For convergent series $\sum_{j=1}^{\infty} \frac{C_{j}^{2}}{j !}$, and any fixed $\varepsilon>0$, let $n_{0}=n_{0}(\varepsilon)$ be such a number that

$$
\sum_{j=m}^{\infty} \frac{C_{j}^{2}}{j !} f^{(* j)}\left(\widehat{\varphi}_{k T}\right)=\sum_{j=m}^{n_{0}} \frac{C_{j}^{2}}{j !} f^{(* j)}\left(\widehat{\varphi}_{k T}\right)+\sum_{j=n_{0}+1}^{\infty} \frac{C_{j}^{2}}{j !} f^{(* j)}\left(\widehat{\varphi}_{k T}\right)=\Sigma_{1}+\Sigma_{2},
$$

and according to (100) $\Sigma_{2} \leq \frac{1}{2 \pi} B_{m} \varepsilon$. 
On the other hand, due to (102), as $T \rightarrow \infty$,

$$
\Sigma_{1}=\sum_{j=m}^{n_{0}} \frac{C_{j}^{2}}{j !} f^{(* j)}\left(\widehat{\varphi}_{k T}\right) \longrightarrow \sum_{j=m}^{n_{0}} \frac{C_{j}^{2}}{j !} f^{(* j)}\left(\varphi_{k}\right) .
$$

Therefore, as $T \longrightarrow \infty$,

$$
4 \pi \sum_{j=m}^{\infty} \frac{C_{j}^{2}}{j !} f^{(* j)}\left(\widehat{\varphi}_{k T}\right) \stackrel{P}{\longrightarrow} 4 \pi \sum_{j=m}^{\infty} \frac{C_{j}^{2}}{j !} f^{(* j)}\left(\varphi_{k T}\right) .
$$

The theorem is then proved due to consistency of $A_{k T}, B_{k T}, k=1, \ldots, N$.

The study of further properties of the proposed covariance matrix $\Gamma$ estimator is a more difficult problem and we address it to subsequent publications

Remark 6 In this paper, we consider the continuous time stochastic processes and observations which is more suitable framework for this classical statistical problem. However, similar results can be obtained for a discrete observations $x_{t}, t \in\{0,1, \ldots, T-1\}$ in the model (11). We only need to replace

$\int_{0}^{T} \ldots$ by $\sum_{t=0}^{T-1} \ldots$ in some steps, and instead of s.d. $f(\lambda), \lambda \in \mathbb{R}$, of the stochastic process with continuous time, we have to use spectral density of discretized process $f_{d}(\lambda)=\sum_{k=-\infty}^{\infty} f(\lambda+2 k \pi)$, $\lambda \in \Lambda=(-\pi, \pi)$, and its convolutions. Also in the integrals in the spectral domain we have to replace $\mathbb{R}^{k}$ into $\Lambda^{k}$.

On the other hand, we can use different numerical procedures to solve a problem related to discrete/continuous observations (for example, smoothing, etc.).

\section{Final Comments}

This paper addresses the problem of consistency, uniqueness and Gaussian limit distribution of the LSE parameter estimate, in the Walker sense, for the non-linear regression model (10), where the regression function has atomic spectral measure. This kind of regression actually constitutes an active research area, due to the existence of several open problems and applications. Note that, although here we have considered the parameter range $\alpha=\min _{l=0, \ldots, \kappa} \alpha_{l}>1 / m$, our conjecture is that the Gaussian limit results hold for

$$
\alpha_{l} \in(0,1), \quad l=0, \ldots, \kappa .
$$

The proof of this conjecture will introduce a general scenario where most of the limit results for random fields with singular spectra (see, for instance, Taqqu (1975, 1979); Dobrushin and Major (1979); Nualart and Peccati (2005); and the references therein) can be obtained as particular cases. New limit results will be required, in the case where the two spectra, the limit regression spectral measure and the spectrum of the Gaussian random field generating the error term, can be overlapped. In this case, different normalizing factors should be considered, leading to different limiting distributions, depending on the common set of spectral singularities co-existing in the regression and error spectra. This case related to the resonance phenomenon will be investigated in subsequent papers, where scaling factors will play a crucial role in the attainment of new limit distributions, and in definition of robust estimates.

\subsection{Acknowledgements}

N.N. Leonenko and M.D. Ruiz-Medina partially supported by grant of the European commission PIRSES-GA-2008-230804 (Maric Curie), projects MTM2009-13393 and MTM2012-32674 of the DGI, and P09-FQM-5052 of the Andalousian CICE, Spain, and the Australian Research Council grants A10024117 and DP 0345577

\section{Appendices}

In the proofs of the Theorems 2 and 3, we use some ideas from Ivanov (1980, 1997, 2010 ), and Ivanov and Leonenko (1989, 2004, 2008, 2009). 


\section{Appendix 1}

Before the proof of Theorem 2, we formulate the following result on the s.v.f.'s.

Lemma 5 Let $\eta \geq 0$ be a given real number, and let function $f(t, s)$ being defined on $(0, \infty) \times(0, \infty)$ such that the integral

$$
\int_{0}^{\beta} \int_{0}^{\beta} f(t, s) \frac{d t d s}{|t-s|^{\eta}}
$$

converges for some $\beta$ from $(0, \infty)$. Let $L$ be a s.v.f. Then for $\eta>0$

$$
\int_{0}^{\beta} \int_{0}^{\beta} f(t, s) L(T|t-s|) \frac{d t d s}{|t-s|^{\eta}} \underset{T \longrightarrow \infty}{\sim} L(T) \int_{0}^{\beta} \int_{0}^{\beta} f(t, s) \frac{d t d s}{|t-s|^{\eta}},
$$

where $a(T) \sim b(T)$ means that $\lim _{T \rightarrow \infty}\{a(T) / b(T)\}=1$.

If $\eta=0$, this relation is valid when the function $L$ is nondecreasing on semi-axis $(0, \infty)$.

The proof of Lemma 1 is similar to the proof of Theorem 2.7 in the book of Seneta (1976).

Proof of Theorem 2. For any $i=1, \ldots, q$, one can write the following identities:

$$
\begin{gathered}
\psi_{T}^{i}(u)-L_{T}^{i}(u)= \\
=\int_{0}^{T} \varepsilon(t) \frac{h_{i}(t, u)}{d_{i T}(\theta)} d t+\int_{0}^{T} H(t ; 0, u) \frac{h_{i}(t, u)}{d_{i T}(\theta)} d t-\int_{0}^{T} \varepsilon(t) \frac{g_{i}(t, \theta)}{d_{i T}(\theta)} d t+\int_{0}^{T} \frac{g_{i}(t, \theta)}{d_{i T}(\theta)} \sum_{l=1}^{q} \frac{g_{l}(t, \theta)}{d_{l T}(\theta)} u_{l} d t \\
=\int_{0}^{T} \varepsilon(t) \frac{H_{i}(t ; u, 0)}{d_{i T}(\theta)} d t+\int_{0}^{T} H(t ; 0, u) \frac{H_{i}(t ; u, 0)}{d_{i T}(\theta)} d t+\int_{0}^{T} \frac{g_{i}(t, \theta)}{d_{i T}(\theta)}\left[H(t ; 0, u)+\sum_{l=1}^{q} \frac{g_{l}(t, \theta)}{d_{l T}(\theta)} u_{l}\right] d t \\
=I_{1}(u)+I_{2}(u)+I_{3}(u) .
\end{gathered}
$$

For fixed $u \in V^{c}(R)$, we get

$$
\begin{aligned}
E I_{1}^{2}(u) & =\int_{0}^{T} \int_{0}^{T} \operatorname{cov}(\varepsilon(t), \varepsilon(s)) \frac{H_{i}(t ; u, 0)}{d_{i T}(\theta)} \frac{H_{i}(s ; u, 0)}{d_{i T}(\theta)} d t d s \leq \\
& \leq \sup _{t \in[0, T]}\left\{\frac{H_{i}^{2}(t, u, 0)}{d_{i T}^{2}(\theta)} \int_{0}^{T} \int_{0}^{T}|E \varepsilon(t) \varepsilon(s)| d t d s\right\} .
\end{aligned}
$$

Under condition B3, and using the finite difference formula, we obtain the following estimates:

$$
\begin{aligned}
\sup _{t \in[0, T]} \frac{\left|H_{i}(t ; u, 0)\right|}{d_{i T}(\theta)} & \leq \sup _{t \in[0, T]}\left\{\sum_{l=1}^{q} \sup _{u \in V^{c}(R)} \frac{\left|h_{i l}(t ; u)\right|}{d_{i T}(\theta) d_{l T}(\theta)}\left|u_{l}\right|\right\} \\
& =\sup _{t \in[0, T]}\left\{\sum_{l=1}^{q} \sup _{u \in V^{c}(R)} \frac{\left|h_{i l}(t ; u)\right|}{d_{i l, T}(\theta)} \cdot \frac{d_{i l, T}(\theta)}{d_{i T}(\theta) d_{l T}(\theta)}\left|u_{l}\right|\right\} \leq R\left(\sum_{l=1}^{q} k_{i l}(R) \tilde{k}_{i l}\right) T^{-1}
\end{aligned}
$$

Then,

$$
E I_{1}^{2}(u) \leq\left(\sum_{l=1}^{q} k_{u}(R) \tilde{k}_{i l}\right)^{2} R^{2} T^{-2} \int_{0}^{T} \int_{0}^{T}|E \varepsilon(t) \varepsilon(s)| d t d s
$$


We will show that

$$
\frac{1}{T^{2}} \int_{0}^{T} \int_{0}^{T}|E \varepsilon(t) \varepsilon(s)| d t d s \longrightarrow 0, \quad T \longrightarrow \infty
$$

From conditions A1-A3,

$$
|E \varepsilon(t) \varepsilon(s)|=\sum_{k=m}^{\infty} \frac{C_{k}^{2}}{k !} B^{k}(t-s) \leq E G^{2}(\xi(0))|B(t-s)|^{m} .
$$

Thus, to prove (106) we need to show that

$$
\frac{1}{T^{2}} \int_{0}^{T} \int_{0}^{T}|B(t-s)|^{m} d t d s \longrightarrow 0, \quad T \longrightarrow \infty .
$$

This is straightforward, for $\alpha \geq 1, \alpha=\min \left(\alpha_{0}, \ldots, \alpha_{\kappa}\right)$. To prove (108) for $\alpha<1$, one can use the inequality:

$$
|B(t)| \leq\left(1+t^{2}\right)^{-\alpha / 2}=B_{0}(t) .
$$

Thus, by the substitutions: $t=t^{*} T, s=s^{*} T$, we have that the left hand side of (108) is bounded by

$$
\begin{aligned}
\frac{1}{T^{2}} \int_{0}^{T} \int_{0}^{T} B_{0}(t-s) d t d s & =\int_{0}^{1} \int_{0}^{1} B_{0}\left(T\left(t^{*}-s^{*}\right)\right) d t^{*} d s^{*} \\
& =\int_{0}^{1} \int_{0}^{1} \frac{L\left(T\left|t^{*}-s^{*}\right|\right)}{T^{\alpha}\left|t^{*}-s^{*}\right|^{\alpha}} d t^{*} d s^{*}=\frac{L(T)}{T^{\alpha}} \int_{0}^{1} \int_{0}^{1} \frac{L\left(T\left|t^{*}-s^{*}\right|\right)}{L(T)} \frac{1}{\left|t^{*}-s^{*}\right|^{\alpha}} d t^{*} d s^{*} \\
& \sim B_{0}(T) \int_{0}^{1} \int_{0}^{1} \frac{d t^{*} d s^{*}}{\left|t^{*}-s^{*}\right|^{\alpha}}=\frac{2}{(1-\alpha)(2-\alpha)} B_{0}(T),
\end{aligned}
$$

where we have applied Lemma [5 with $f(t, s)=\left|t^{*}-s^{*}\right|^{-\alpha}$. For $\eta>0$ one can take any number such that $\alpha+\eta<1$. From (108), we therefore obtain that $I_{1}(u) \longrightarrow 0$, as $T \longrightarrow \infty$, in probability, pointwise, for $u \in V^{c}(R)$.

On the other hand,

$$
\begin{aligned}
P\left\{\sup _{\left\|u_{1}-u_{2}\right\| \leq h}\left|I_{1}\left(u_{1}\right)-I_{1}\left(u_{2}\right)\right|>r\right\} & \leq r^{-1} E \sup _{\left\|u_{1}-u_{2}\right\| \leq h}\left|\int_{0}^{T} \varepsilon(t) \frac{H_{i}\left(t ; u_{1}, u_{2}\right)}{d_{i T}(\theta)} d t\right| \\
& \leq r^{-1} \sup _{\left\|u_{1}-u_{2}\right\| \leq h \sup _{t \in[0, T]}} \frac{\left|H_{i}\left(t ; u_{1}, u_{2}\right)\right|}{d_{i T}(\theta)} E|\varepsilon(0)| T .
\end{aligned}
$$

Under B3, we have

$\sup _{\left\|u_{1}-u_{2}\right\| \leq h} \sup _{t \in[0, T]} \frac{\left|H_{i}\left(t ; u_{1}, u_{2}\right)\right|}{d_{i T}(\theta)} \leq h \sup _{t \in[0, T]}\left\{\sum_{l=1}^{q} \sup _{u \in V^{C}(R)} \frac{\left|h_{i l}(t ; u)\right|}{d_{i l, T}(\theta)} \cdot \frac{d_{i l, T}(\theta)}{d_{i T}(\theta) d_{l T}(\theta)}\right\} \leq h\left(\sum_{l=1}^{q} k_{i l}(R) \tilde{k}_{i l}\right) T^{-1}$.

From (111), we obtain

$$
P\left\{\sup _{\left\|u_{1}-u_{2}\right\| \leq h}\left|I_{1}\left(u_{1}\right)-I_{1}\left(u_{2}\right)\right|>r\right\} \leq k_{1} r^{-1} h
$$

where

$$
k_{1}=\left(\sum_{l=1}^{q} k_{i l}(R) \tilde{k}_{i l}\right) E|\varepsilon(0)| .
$$


Let $N_{h}$ be a finite $h$-net of the ball $V^{c}(R)$. Then

$$
\sup _{u \in V^{c}(R)}\left|I_{1}(u)\right| \leq \sup _{\left\|u_{1}-u_{2}\right\| \leq h}\left|I_{1}\left(u_{1}\right)-I_{1}\left(u_{2}\right)\right|+\max _{u \in N_{h}}\left|I_{1}(u)\right| .
$$

From (110) and (111), and for any $r>0$,

$$
P\left\{\sup _{u \in V^{c}(R)}\left|I_{1}(u)\right|>r\right\} \leq 2 k_{1} r^{-1} h+P\left\{\max _{u \in N_{h}}\left|I_{1}(u)\right|>\frac{r}{2}\right\} .
$$

For $\epsilon>0$, we have $h=\frac{\epsilon r}{4 k_{1}}$. Since $I_{1}(u) \stackrel{P}{\longrightarrow} 0$ pointwise, for $T>T_{0}$,

$$
P\left\{\max _{u \in N_{\frac{\epsilon r}{4 k_{1}}}}\left|I_{1}(u)\right|>\frac{r}{2}\right\} \leq \frac{\epsilon}{2}
$$

and

$$
P\left\{\sup _{u \in V^{c}(R)}\left|I_{1}(u)\right|>r\right\} \leq \epsilon .
$$

Thus, $I_{1}(u) \longrightarrow 0$, as $T \longrightarrow \infty$, in probability, uniformly for $u \in V^{c}(R)$.

From B3 and Cauchy-Schwartz inequality, applying the Lagrange formula

$$
\begin{aligned}
\sup _{u \in V^{c}(R)} \sup _{t \in[0, T]}|H(t ; 0, u)| & =\sup _{u \in V^{c}(R)} \sup _{t \in[0, T]}\left|\sum_{i=1}^{q} \frac{h_{i}\left(t ; u_{t}^{*}\right)}{d_{i T}(\theta)} u_{i}\right| \\
& \leq \sup _{u \in V^{c}(R)}\|u\|\left[\sup _{t \in[0, T]} \sum_{i=1}^{q}\left(\frac{h_{i}\left(t ; u_{t}^{*}\right)}{d_{i T}(\theta)}\right)^{2}\right]^{1 / 2} \leq\|k(R)\| R T^{-1 / 2}
\end{aligned}
$$

where $k(R)=\left(k^{1}(R), \ldots, k^{q}(R)\right)$.

From (104) and (115), we have

$$
\begin{aligned}
\sup _{u \in V^{c}(R)}\left|I_{2}(u)\right| & =\sup _{u \in V^{c}(R)}\left|\int_{0}^{T} H(t ; 0, u) \frac{H_{i}(t ; u, 0)}{d_{i T}(\theta)} d t\right| \\
& \leq T \sup _{u \in V^{c}(R)} \sup _{t \in[0, T]}\left|H(t ; 0, u) \frac{H_{i}(t ; u, 0)}{d_{i T}(\theta)}\right| \leq\|k(R)\| R^{2} T^{-1 / 2}\left(\sum_{l=1}^{q} k_{i l}(R) \tilde{k}_{i l}\right) .
\end{aligned}
$$

Thus, $I_{2}(u) \longrightarrow 0$, as $T \longrightarrow \infty$, uniformly for $u \in V^{c}(R)$.

Now, $I_{3}(u)$ can be written as

$$
\begin{aligned}
I_{3}(u) & =\int_{0}^{T} \frac{g_{i}(t, \theta)}{d_{i T}(\theta)}\left[H(t ; 0, u)+\sum_{l=1}^{q} \frac{g_{l}(t, \theta)}{d_{l T}(\theta)} u_{l}\right] d t=-\frac{1}{2} \int_{0}^{T} \frac{g_{i}(t, \theta)}{d_{i T}(\theta)} \sum_{l, j=1}^{q} \frac{h_{l j}\left(t ; u_{T}^{*}\right)}{d_{l T}(\theta) d_{j T}(\theta)} u_{l} u_{j} d t \\
& =-\frac{1}{2} \sum_{l, j=1}^{q}\left(\int_{0}^{T} \frac{h_{l j}\left(t ; u_{T}^{*}\right)}{d_{l T}(\theta) d_{j T}(\theta)} \frac{g_{i}(t, \theta)}{d_{i T}(\theta)} d t\right) u_{l} u_{j}, \quad u_{T}^{*} \in V^{c}(R) .
\end{aligned}
$$

From B3, applying Cauchy-Schwartz inequality:

$\sup _{u \in V^{c}(R)}\left|I_{3}(u)\right| \leq \frac{T}{2} k^{i}(R)\left(\sum_{l, j=1}^{q} k_{j l}(R) \tilde{k}_{j l}\left|u_{j}\right|\left|u_{l}\right|\right) T^{-3 / 2} \leq \frac{q k^{i}(R)}{2} \max _{1, \leq j, l \leq q}\left\{k_{j l}(R) \tilde{k}_{j l}\right\}\|u\|^{2} T^{-1 / 2}$.

Thus, $I_{3}(u) \longrightarrow 0$, as $T \longrightarrow \infty$, uniformly for $u \in V^{c}(R)$. Theorem 2 then follows. 


\section{Appendix 2}

The proof of the Theorem 3 is derived in this appendix.

We consider a Hessian $\mathcal{H}_{T}(w)=\left(\mathcal{H}_{T}^{i l}(w)\right)_{i, l=1}^{q}$,

$$
\begin{aligned}
\mathcal{H}_{T}^{i l}(w)= & \frac{\partial^{2}}{\partial w_{i} \partial w_{l}}\left(\frac{1}{2 T} Q_{T}\left(\theta+T^{1 / 2} d_{T}^{-1}(\theta) w\right)\right) \\
= & T^{-1} \int_{0}^{T}\left([x(t)-f(t, w)] \frac{-f_{i l}(t, w)}{d_{i T}(\theta) d_{l T}(\theta)} T+\frac{f_{i}(t, w) f_{l}(t, w)}{d_{i T}(\theta) d_{l T}(\theta)} T\right) d t \\
= & \int_{0}^{T}[f(t, 0)+\varepsilon(t)-f(t, w)] \frac{-f_{i l}(t, w)}{d_{i T}(\theta) d_{l T}(\theta)} d t+\int_{0}^{T} \frac{f_{i}(t, w) f_{l}(t, w)}{d_{i T}(\theta) d_{l T}(\theta)} d t \\
= & \int_{0}^{T} F(t ; w, 0) \frac{f_{i l}(t, w)}{d_{i T}(\theta) d_{l T}(\theta)} d t-\int_{0}^{T} \varepsilon(t) \frac{f_{i l}(t, w)}{d_{i T}(\theta) d_{l T}(\theta)} d t \\
& +\int_{0}^{T} \frac{\left(f_{i}(t, w)-f_{i}(t, 0)+f_{i}(t, 0)\right)\left(f_{l}(t, w)-f_{l}(t, 0)+f_{l}(t, 0)\right)}{d_{i T}(\theta) d_{l T}(\theta)} d t \\
= & I_{1}^{i l}(w)+I_{2}^{i l}(w)+\int_{0}^{T} \frac{\left(f_{i}(t, w)-f_{i}(t, 0)\right)\left(f_{l}(t, w)-f_{l}(t, 0)\right)}{d_{i T}(\theta) d_{l T}(\theta)} \\
& +\int_{0}^{T} \frac{\left(f_{i}(t, w)-f_{i}(t, 0)\right) f_{l}(t, 0)}{d_{i T}(\theta) d_{l T}(\theta)} d t+\int_{0}^{T} \frac{\left.f_{i}(t, 0)\right)\left(f_{l}(t, w)-f_{l}(t, 0)\right)}{d_{i T}(\theta) d_{l T}(\theta)} d t+\int_{0}^{T} \frac{\left.f_{i}(t, 0)\right) f_{l}(t, 0)}{d_{i T}(\theta) d_{l T}(\theta)} d t \\
= & I_{1}^{i l}(w)+I_{2}^{i l}(w)+I_{3}^{i l}(w)+I_{4}^{i l}(w)+I_{5}^{i l}(w)+J_{i l, T}(\theta), \quad i, l=1, \ldots, q .
\end{aligned}
$$

From the inequality

$$
\left|\lambda_{\min }\left(\mathcal{H}_{T}(w)\right)-\lambda_{\min }\left(J_{T}\left(\theta^{0}\right)\right)\right| \leq q \max _{1 \leq i, l \leq q}\left|\mathcal{H}_{T}^{i l}(w)-J_{T}^{i l}(\theta)\right|
$$

(see, Wilkinson (1965), p.103), we have

$$
\max _{1 \leq i, l \leq q}\left|\mathcal{H}_{T}^{i l}(w)-J_{T}^{i l}(\theta)\right| \leq \sum_{m=1}^{5} \max _{1 \leq i, l \leq q}\left|I_{m}^{i l}(w)\right| .
$$

Applying Cauchy-Schwartz inequality, and the Lagrange formula, for $\|w\| \leq r_{0}$ :

$$
\begin{aligned}
\left|I_{1}(w)\right| & =\left|\int_{0}^{T} F(t ; w, 0) \frac{f_{i l}(t, w)}{d_{i T}(\theta) d_{l T}(\theta)} d t\right| \leq T \sup _{t \in[0, T]}\left|F(t ; w, 0) \frac{f_{i l}(t, w)}{d_{i T}(\theta) d_{l T}(\theta)}\right| \\
& \leq T \hat{k}^{i l} \tilde{k}^{i l} T^{-1} \sup _{t \in[0, T]}|F(t ; w, 0)| \leq \hat{k}^{i l} \tilde{k}^{i l} \sup _{t \in[0, T]}|f(t, w)-f(t, 0)| \\
& =\hat{k}^{i l} \tilde{k}^{i l} \sup _{t \in[0, T]}\left|T^{1 / 2} \sum_{l=1}^{q} \frac{f_{l}\left(t, w_{T}^{*}\right)}{d_{l T}(\theta)} w_{l}\right| \\
& \leq \hat{k}^{i l} \tilde{k}^{i l} T^{1 / 2} \sup _{t \in[0, T]}\left(\sum_{l=1}^{q}\left(\frac{f_{l}\left(t, w_{T}^{*}\right)}{d_{l T}(\theta)}\right)^{2}\right)^{1 / 2}\|w\| \leq\|\hat{k}\| \hat{k}^{i l} \tilde{k}^{i l}\|w\|, \hat{k}=\left(\hat{k}_{1}, \ldots, \hat{k}_{q}\right) .
\end{aligned}
$$

We now consider

$\left|I_{2}(w)\right|=\left|\int_{0}^{T} \varepsilon(t) \frac{f_{i l}(t, w)}{d_{i T}(\theta) d_{l T}(\theta)} d t\right|=\left|\int_{0}^{T} \varepsilon(t) \frac{F_{i l}(t ; w, 0)}{d_{i T}(\theta) d_{l T}(\theta)} d t+\int_{0}^{T} \varepsilon(t) \frac{f_{i l}(t, 0)}{d_{i T}(\theta) d_{l T}(\theta)} d t\right| \leq\left|I_{6}(w)\right|+\left|I_{7}(w)\right|$. 
From B5, we have

$$
\begin{gathered}
\left|I_{6}(w)\right|=\left|\int_{0}^{T} \varepsilon(t) \frac{F_{i l}(t ; w, 0)}{d_{i T}(\theta) d_{l T}(\theta)} d t\right| \leq\left(\frac{1}{T} \int_{0}^{T} \varepsilon^{2}(t)\right)^{1 / 2}\left(T \frac{\Phi_{i l, T}(w, 0)}{d_{i T}^{2}(\theta) d_{l T}^{2}(\theta)}\right)^{1 / 2} \leq\left(\frac{1}{T} \int_{0}^{T} \varepsilon^{2}(t)\right)^{1 / 2}\left(\hat{k}_{i l}\right)^{1 / 2}\|w\| . \\
\text { Then, }\left(\frac{1}{T} \int_{0}^{T} \varepsilon^{2}(t)\right)^{1 / 2}=\left(\frac{1}{T} \int_{0}^{T}\left(\varepsilon^{2}(t)-E \varepsilon^{2}(0)\right) d t+E \varepsilon^{2}(0)\right)^{1 / 2}=\left(\xi_{T}+E \varepsilon^{2}(0)\right)^{1 / 2} \\
E \xi_{T}^{2}=T^{-2} \int_{0}^{T} \int_{0}^{T}\left(E \varepsilon^{2}(t) \varepsilon^{2}(s)-\left(E \varepsilon^{2}(0)\right)^{2}\right) d t d s .
\end{gathered}
$$

We will prove that

$$
T^{-2} \int_{0}^{T} \int_{0}^{T} E \varepsilon^{2}(t) \varepsilon^{2}(s) d t d s \longrightarrow\left(E \varepsilon^{2}(0)\right)^{2}, T \longrightarrow \infty
$$

Under A2, the function $G^{2}(x) \in L_{2}\left(\mathbb{R}^{1}, \phi(x) d x\right), G^{2}(x)=\sum_{k=0}^{\infty} \frac{d_{k}}{k !} H_{k}(x), d_{k}=\int_{-\infty}^{\infty} G^{2}(x) H_{k}(x) \phi(x) d x, \quad k \geq$ 0. Thus,

$$
E \varepsilon^{2}(t) \varepsilon^{2}(s)-\left(E \varepsilon^{2}(0)\right)^{2}=\sum_{k=0}^{\infty} \frac{d_{k}^{2}}{k !} B^{k}(t-s)-\left(E \varepsilon^{2}(0)\right)^{2}=\sum_{k=1}^{\infty} \frac{d_{k}^{2}}{k !} B^{k}(t-s) \leq|B(t-s)| \sum_{k=1}^{\infty} \frac{d_{k}^{2}}{k !} .
$$

Since

$$
\sum_{k=1}^{\infty} \frac{d_{k}^{2}}{k !}=E G^{4}(\xi(0))-\left[E G^{2}(\xi(0))\right]^{2}=D E G^{2}(\xi(0))<\infty
$$

we have from (123)-(124) as $T \longrightarrow \infty$,

$$
T^{-2} \int_{0}^{T} \int_{0}^{T}\left(E \varepsilon^{2}(t) \varepsilon^{2}(s)-\left(E \varepsilon^{2}(0)\right)^{2}\right) d t d s \leq D E G^{2}(\xi(0)) T^{-2} \int_{0}^{T} \int_{0}^{T}|B(t-s)| d t d s \longrightarrow 0,
$$

as it was already proven in Appendix 1.

We will write

$$
\xi_{T}=o_{p}^{(1)}(1), \quad o_{p}^{(1)}(1) \longrightarrow^{P} 0, \quad \text { as } T \longrightarrow \infty .
$$

From B3 and B5,

$$
\begin{aligned}
E\left|I_{7}(w)\right|^{2} & =E\left|\int_{0}^{T} \varepsilon(t) \frac{f_{i l}(t ; 0)}{d_{i T}(\theta) d_{l T}(\theta)} d t\right|^{2} \leq \int_{0}^{T} \int_{0}^{T}|E \varepsilon(t) \varepsilon(s)|\left(\sup _{t \in[0, T]} \frac{f_{i l}(t ; 0)}{d_{i T}(\theta) d_{l T}(\theta)}\right)^{2} \\
& \leq\left(\hat{k}^{i l} \tilde{k}^{i l}\right)^{2} D E G(\xi(0)) T^{-2} \int_{0}^{T} \int_{0}^{T}|B(t-s)|^{m} d t d s,
\end{aligned}
$$

and

$$
\left|I_{7}(w)\right|=o_{p}^{(2)}(1), \quad o_{p}^{(2)}(1) \longrightarrow^{P} 0, \quad \text { as } \quad T \longrightarrow \infty .
$$


We can continue as follow

$$
\begin{aligned}
\left|I_{3}(w)\right| & =\left|\int_{0}^{T} \frac{\left(f_{i}(t, w)-f_{i}(t, 0)\right)\left(f_{l}(t, w)-f_{l}(t, 0)\right)}{d_{i T}(\theta) d_{l T}(\theta)} d t\right| \\
& \leq T \sum_{j=1}^{q} \sum_{s=1}^{q} \int_{0}^{T} \frac{\left|f_{i j}\left(t, w_{T}^{*}\right)\right|}{d_{i T}(\theta) d_{j T}(\theta)} \frac{\left|f_{l s}\left(t, w_{T}^{*}\right)\right|}{d_{s T}(\theta) d_{l T}(\theta)} d t\left|w_{j}\right|\left|w_{s}\right| \\
& \leq T \sum_{j=1}^{q} \sum_{s=1}^{q} \int_{0}^{T} \frac{\left|f_{i j}\left(t, w_{T}^{*}\right)\right|}{d_{i j, T}(\theta)} \frac{d_{i j, T}(\theta)}{d_{i T}(\theta) d_{j T}(\theta)} \frac{\left|f_{l s}\left(t, w_{T}^{*}\right)\right|}{d_{l s, T}(\theta)} \frac{d_{l s, T}(\theta)}{d_{s T}(\theta) d_{l T}(\theta)} d t\left|w_{j}\right|\left|w_{s}\right| \\
& \leq\left(\sum_{s=1}^{q}\left(\hat{k}^{l s} \tilde{k}^{l s}\right)^{2}\right)^{1 / 2} \cdot\left(\sum_{j=1}^{q}\left(\hat{k}^{i j} \tilde{k}^{i j}\right)^{2}\right)^{1 / 2}\|w\|^{2} .
\end{aligned}
$$

From B3 and B5, we obtain

$$
\begin{aligned}
\left|I_{4}(w)\right| & =\left|\int_{0}^{T} \frac{\left(f_{i}(t, w)-f_{i}(t, 0) f_{l}(t, 0)\right.}{d_{i T}(\theta) d_{l T}(\theta)} d t\right| \\
& \leq T^{1 / 2} \sum_{j=1}^{q}\left|w_{j}\right| \int_{0}^{T} \frac{\left|f_{i j}\left(t, w_{T}^{*}\right)\right|}{d_{i j, T}(\theta)} \frac{d_{i j, T}(\theta)}{d_{i T}(\theta) d_{j T}(\theta)} \frac{\left|f_{l}(t, 0)\right|}{d_{l T}(\theta)} d t \\
& \leq T^{3 / 2} \sum_{j=1}^{q}\left|w_{j}\right| \sup _{t \in[0, T]}\left\{\frac{\left|f_{i j}\left(t, w_{T}^{*}\right)\right|}{d_{i j, T}(\theta)} \frac{d_{i j, T}(\theta)}{d_{i T}(\theta) d_{j T}(\theta)} \frac{\left|f_{l}(t, 0)\right|}{d_{l T}(\theta)}\right\} \\
& \leq \hat{k}^{l}\left(\sum_{j=1}^{q}\left(\hat{k}^{i j} \tilde{k}^{i j}\right)^{2}\right)^{1 / 2}\|w\| .
\end{aligned}
$$

Similarly for $I_{5}(w)$, we have

$$
\left|I_{5}(w)\right| \leq \hat{k}^{i}\left(\sum_{j=1}^{q}\left(\hat{k}^{l j} \tilde{k}^{l j}\right)^{2}\right)^{1 / 2}\|w\| .
$$

From (119)-(129), we get

$$
\begin{aligned}
& \left|\lambda_{\min }\left(\mathcal{H}_{T}(w)\right)-\lambda_{\min }\left(J_{T}\left(\theta^{0}\right)\right)\right| \leq q \max _{1 \leq i, l \leq q}\left\{\|\hat{k}\| \hat{k}^{i l} \tilde{k}^{i l}\|w\|+\left[\left(o_{p}^{(1)}(1)+1\right)^{1 / 2}\left(\hat{k}_{i j}\right)^{1 / 2}\|w\|+o_{p}^{(2)}(1)\right]\right. \\
& \left.+\left(\sum_{s=1}^{q}\left(\hat{k}^{l s} \tilde{k}^{l s}\right)^{2}\right)^{1 / 2}\left(\sum_{j=1}^{q}\left(\hat{k}^{i j} \tilde{k}^{i j}\right)^{2}\right)^{1 / 2}\|w\|^{2}+\hat{k}^{l}\left(\sum_{j=1}^{q}\left(\hat{k}^{i j} \tilde{k}^{i j}\right)^{2}\right)^{1 / 2}\|w\|+\hat{k}^{i}\left(\sum_{j=1}^{q}\left(\hat{k}^{l j} \tilde{k}^{l j}\right)^{2}\right)^{1 / 2}\|w\|\right\} .
\end{aligned}
$$

We substitute the estimate (130) into the normed LSE $\hat{w}_{T}$, and, by B4 (from which it is follows that $J_{T}\left(\theta^{0}\right)>0$, with the minimal eigenvalue $\lambda_{\min }\left(J_{T}\left(\theta^{0}\right)\right) \geq \lambda_{*}$, for some $r>0$, we introduce the event

$$
\begin{aligned}
\Omega_{1} \cap \Omega_{2} \cap \Omega_{3}= & \left\{\left|o_{p}^{(1)}(1)\right| \leq r,\left|o_{p}^{(2)}(1)\right| \leq r,\left\|\hat{w}_{T}\right\| \leq r\right\} \subset\left\{\left|\lambda_{\min }\left(\mathcal{H}_{T}\left(\hat{w}_{T}\right)\right)-\lambda_{\min }\left(J_{T}\left(\theta^{0}\right)\right)\right| \leq \frac{\lambda_{*}}{2}\right\} \\
& =\left\{\lambda _ { \operatorname { m i n } } \left(J_{T}\left(\theta^{0}\right)-\frac{\lambda_{*}}{2} \leq \lambda_{\min }\left(\mathcal{H}_{T}\left(\hat{w}_{T}\right)\right) \leq \lambda_{\min }\left(J_{T}\left(\theta^{0}\right)+\frac{\lambda_{*}}{2}\right\}\right.\right. \\
& \subset\left\{\lambda_{\min }\left(\mathcal{H}_{T}\left(\hat{w}_{T}\right)\right) \geq \lambda_{\min }\left(J_{T}\left(\theta^{0}\right)-\frac{\lambda_{*}}{2}\right\} \subset\left\{\lambda_{\min }\left(\mathcal{H}_{T}\left(\hat{w}_{T}\right)\right) \geq \frac{\lambda_{*}}{2}\right\} .\right.
\end{aligned}
$$


We then have

$$
P\left\{\overline{\Omega_{1} \cap \Omega_{2} \cap \Omega_{3}}\right\} \leq P\left\{\left|o_{p}^{(1)}(1)\right|>r\right\}+P\left\{\left|o_{p}^{(2)}(1)\right|>r\right\}+P\left\{\left\|\hat{w}_{T}\right\|>r\right\} .
$$

For any $\epsilon>0$ and $T>T_{0}$, we obtain $P\left\{\left|o_{p}^{(1)}(1)\right|>r\right\} \leq \frac{\epsilon}{3}, P\left\{\left|o_{p}^{(2)}(1)\right|>r\right\} \leq \frac{\epsilon}{3}$. Note that if $T>T_{0}$, then, $P\left\{\left\|\hat{w}_{T}\right\|>r\right\} \leq \frac{\epsilon}{3}$, and hence,

$$
P\left\{\left|o_{p}^{(1)}(1)\right|>r\right\}+P\left\{\left|o_{p}^{(2)}(1)\right|>r\right\}+P\left\{\left\|\hat{w}_{T}\right\|>r\right\} \leq \frac{\epsilon}{3}+\frac{\epsilon}{3}+\frac{\epsilon}{3}=\epsilon .
$$

Therefore, for $T>T_{0}, P\left\{\Omega_{1} \cap \Omega_{2} \cap \Omega_{3}\right\}>1-\epsilon$. This means that the normed $\hat{w}_{T}$ is the unique solution of the equation (55), with probability tending to 1 as $T \longrightarrow \infty$, since the matrix $\mathcal{H}_{T}\left(\hat{w}_{T}\right)$ is positive definite and the functional $Q_{T}(\theta, \omega)$ has unique minimum at the point $\hat{w}_{T}$. Thus, Theorem 3 is proven.

\section{References}

[1] Anh, V.V., Knopova, V.P. and Leonenko, N.N. (2004). Continuous-time stochastic processes with cyclical long-range dependence. Australian and NZ J. of Statistics 46, 275-296.

[2] Arcones, M. A. (1994). Limit theorems for nonlinear functionals of a stationary Gaussian sequence of vectors. Ann. Probab. 22, 2242-2274.

[3] Arcones, M. A. (2000). Distributional limit theorems over a stationary Gaussian sequence of random vectors. Stochastic Process. Appl. 88, 135-159.

[4] Avram, F. (1992). Generalized Szegö Theorems and asymptotics of cumulants by graphical methods. Transactions of the American Mathematical Society 330, 637-649.

[5] Avram, F. and Brown, L. A. (1989). Generalized Hölder Inequality and a Generalized Szegö Theorem. Proceedings of the American Mathematical Society 107, 687-695.

[6] Avram, F. and Fox, R. (1992). Central limit theorems for sums of Wick products of stationary sequences. Transactions of the American Mathematical Society 330,651-663.

[7] Avram, F. Leonenko N.N. and Sakhno, L. (2010). On Szegö type limit theorem, the Holder -Young-Brascamp-Lieb inequality, and asymptotic theory of integrals and quadratic forms of stationary fields. ESAIM: Probability and Statistics, 14, 210-255.

[8] Bhattacharya, R.N. and Ranga Rao, R. (1976). Normal Approximation and Asymptotic Expansions, Wiley, New Yoark

[9] Berman, S.M. (1992). A central limit theorem for the renormalized self-intersection local time of a stationary vector Gaussian process. Annals of Probability 20, 61-81.

[10] Breuer, P. and Major, P. (1983). Central limit theorems for nonlinear functionals of Gaussian fields. J. Multiv. Anal. 13, 425-441.

[11] Dobrushin R.L. and Major, P. (1979). Non-central limit theorem for non-linear functionals of Gaussian fields. Z Wahrsch. Verw. Geb. 50, 1-28.

[12] Donoghue, W.J. (1969). Distributions and Fourier Transforms. Academic Press, New York.

[13] Doukhan, P., Oppenheim, G. and Taqqu, M.S. (2003). Theory and Applications of Long-range Dependence. Birkhäuser, Boston.

[14] Gradshteyn, I.S. and Ryzhik, I.M.(2000) Tables of Integrals, Series and Products, sixth ed., Academic Press, San Diego.

[15] Grenander, U. and Rosenblatt, M. (1984). Statistical Analysis of Stationary Time Series. Chelsea Publ. Company, New York. 
[16] Hannan, E.(1973) The asymptotic theory of linear time-series models. J. Appl. Probab., 10, $510-519$

[17] Haye, O.M. (2002). Asymptotic behavior of the empirical processes for Gaussian data presented seasonal long-memory. ESAIM: Probability and Statistics 6, 293-309.

[18] Haye, O.M. and Phillipe, A. (2003). A noncentral limit theorem for the empirical processes of linear sequences with seasonal long memory. Mathematical Methods of Statistics 12, 329-357.

[19] Haye, O.M. and Viano M.-C. (2002). Limit theorems under seasonal long-memory. In Theory and Applications of Long-Range Dependence. Doukhan, P et al. (Eds.). Birkhauser, Boston, 101110.

[20] Ho, H.C. and Hsing, T. (1997). Limit theorems for functionals of moving average. Ann.Probab. 25, 1636-1669.

[21] Holevo, A.S. (1976). On the asymptotic efficient regression estimates in the case of degenerate spectrum. Theory Probab. Appl. 21, 324-333.

[22] Ibragimov, I.A. and Rozanov, Yu.A. (1980). Gaussian Random Processes. Springer-Verlag, New York.

[23] Ivanov, A.V. (1980). A solution of the problem of detecting hidden periodicities. Theory Probability and Math.Stat. 20, 51-68.

[24] Ivanov, A.V. (1997). Asymptotic Theory of Nonlinear Regression. Kluwer Academic Publishers. Dordrecht.

[25] Ivanov, A.V (2010) Consistency of the least squares estimator of the amplitudes and angular frequencies of the sum of harmonic oscillations in models with strong dependence. Theory Probab. Math. Statist. 80, 61-69

[26] Ivanov, A.V. and Leonenko, N.N. (1989). Statistical Analysis of Random Fields. Kluwer Academic Publishers, Dordrecht.

[27] Ivanov, A.V. and Leonenko, N.N. (2004). Asymptotic theory for non-linear regression with long-range dependence. Mathematical Methods of Statistics 13, 153-178.

[28] Ivanov, A.V. and Leonenko, N.N. (2008). Semiparametric analysis of long-range dependence in nonlinear regression, J. Statist. Plann. Inference 138, 1733-1753.

[29] Ivanov, A.V. and Leonenko, N.N. (2009) Robust estimators in non-linear regression models with long-range dependence. Optimal design and related areas in optimization and statistics, 193-221, Springer Optim. Appl., 28, Springer, New York.

[30] Ivanov, A.V. and Orlovski I.V. (2008) Asymptotic normality of $\$ M \$ M-e s t i m a t e s$ in the classical nonlinear regression model, Ukrainian Math. J. 60, no. 11, 1716-1739.

[31] Koul H. (1996) Asymptotics of M-estimations in non-linear regression with long-range dependence errors. In: Proc. Athens Conf. Appl. Probab. and Time Ser. Analysis (P.M. Robinson and M. Rosenblatt, Eds.) Springer Verlag Lecture Notes in Statistics, II, 272-291.

[32] Koul H and Baillie, R.T. (2003) Asymptotics of M-estimators in non-linear regression models with long-memory designs. Statistics and Probability Letters, 61, 237-252.

[33] Leonenko, N.N. (1999). Limit Theorems for Random Fields with Singular Spectrum. Kluwer Academic Publishers, Dordrecht.

[34] Leonenko, N.N. and Taufer, E. (2006). Weak convergence of weighted quadratic functionals of stationary long memory processes to Rosenblatt-type distributions. J. Statist. Planning and Inference 136, 1220-1236. 
[35] Milnor, J.W. (1965) Topology from the Differentiable Viewpoint. Princeton University Press. Princeton, NJ.

[36] Mukhergee, K (2000) Linearization of randomly weighted empiricals under long range dependence with applications to nonlinear regression quantiles. Economic Theory, 16, 301-323

[37] Nualart, D. (1995). The Malliavin Calculus and Related Topics. Springer-Verlag, New York.

[38] Nualart, D. and Peccati, G. (2005). Central limit theorems for sequences of multiple stochastic integrals. The Annals of Probability 33, 177-193.

[39] Oppenheim, G., Ould H.M. and Viano M.-C. (2002). Long memory with seasonal effects. Statitical Inference for Stochastic Processes 3, 53-68.

[40] Peccati, G. (2009). Stein's method, Malliavin calculus and infinite-dimensional Gaussian analysis. Lecture Notes (www.glocities.com/giovannipeccati).

[41] Peccati, G. and Taqqu, M.S. (2010). Wiener Chaos: Moments, Cumulants and Diagrams. Springer, Berlin.

[42] Peccati, G. and Tudor, C.A. (2004). Gaussian limits for vector-valued multiple stochastic integrals. Séminaire de Probabilités XXXVIII, 247-262.

[43] Pollard, D. and Radchenko, P. (2006) Nonlinear least-squares estimation. J. Multiv. Anal., 97, 548-562.

[44] Quinn, B.G. And Hannan, E.J. (2001). The Estimation and Tracking of Frequency. Cambridge University Press. New York.

[45] Robinson, P.M. And Hidalgo, F.J. (1997) Time series regression with long-range dependence. Ann. Statist., 25, 77-104

[46] Rosenblatt, M. (1976). Fractional integrals of stationary processes and the central limit theorem. J. Appl. Prob. 13, 723-732.

[47] Rosenblatt, M. (1981). Limit theorems for Fourier transforms of functionals of Gaussian sequences. Z. Wahrsch. Verw. Gebiete 55, 123-132.

[48] Rosenblatt, M. (1987). Scale renormalization and random solutions of Burgers equation. J. Appl. Prob. 24, 328-338.

[49] Seneta, E (1976) Regularly Varying Functions. Lecture Notes in Mathematics. 508, SpringerVerlag, Berlin-New York.

[50] Skouras, K (2000) Strong consistency in nonlinear regression models. Ann. Statist., 28, 871-879

[51] TAQQU, M.S. (1975). Weak convergence to fractional Brownian motion and to the Rosenblatt process. Z. Wahrsch. Verw. Gebiete 31, 287-302.

[52] TAQQU M.S. (1979). Convergence of integrated processes of arbitrary Hermite rank. Z. Wahrsch. Verw. Gebiete 50, 53-83.

[53] Viano, M.-C., Deniau, Cl. and Oppenheim, G. (1995). Long-range dependence and mixing for discrete time fractional processes. J. Time Series Analysis 16, 323-338.

[54] Walker, A.M. (1973) On the estimation of a harmonic component in a time series with stationary dependent residuals, Adv. Appl. Probab., 5, 217-241.

[55] Whittle, P. (1952). The simultaneous estimation of a time series harmonic components and covariance structure. Trabajos Estadística 3, 43-57.

[56] Wilkinson, J.H. (1965). The Algebraic Eigenvalue Problem. Clarendon Press, Oxford. 
[57] Yajima, Y. (1988). On estimation of a regression model with long-memory stationary errors. Ann. Statist. 16, 791-807.

[58] Yajima, Y. (1991). Asymptotic properties of the LSE in a regression model with long-memory stationary errors. Ann. Statist. 19, 158-177. 